Draft VERSiOn MAY 7, 2018

Preprint typeset using $\mathrm{LATEX}_{\mathrm{E}}$ style emulateapj v. 12/16/11

\title{
ELUCID V. LIGHTING DARK MATTER HALOS WITH GALAXIES
}

\author{
Xiaohu Yang ${ }^{1,2}$, Youcai Zhang ${ }^{3}$, Huiyuan Wang ${ }^{4,5}$, Chengze Liu ${ }^{1}$, Tianhuan Lu ${ }^{6}$, Shijie Li ${ }^{1}$, Feng Shi $^{3}$, Y.P. \\ Jing $^{1,2}$, H.J. Mo ${ }^{7,8}$, Frank C. VAn den Bosch ${ }^{9}$, Xi Kang ${ }^{10}$, Weiguang Cui ${ }^{11}$, Hong Guo ${ }^{3}$, Guoliang Li ${ }^{9}$, S.H. Lim ${ }^{7}$, \\ Yi Lu ${ }^{3}$, Wentao Luo ${ }^{1}$, Chengliang $\mathrm{Wei}^{9}$, Lei Yang ${ }^{1}$ \\ Draft version May 7, 2018
}

\begin{abstract}
In a recent study, using the distribution of galaxies in the north galactic pole of SDSS DR7 region enclosed in a $500 h^{-1} \mathrm{Mpc}$ box, we carried out our ELUCID simulation (Wang et al. 2016, ELUCID III). Here we light the dark matter halos and subhalos in the reconstructed region in the simulation with galaxies in the SDSS observations using a novel neighborhood abundance matching method. Before we make use of thus established galaxy-subhalo connections in the ELUCID simulation to evaluate galaxy formation models, we set out to explore the reliability of such a link. For this purpose, we focus on the following a few aspects of galaxies: (1) the central-subhalo luminosity and mass relations; (2) the satellite fraction of galaxies; (3) the conditional luminosity function (CLF) and conditional stellar mass function (CSMF) of galaxies; and (4) the cross correlation functions between galaxies and the dark matter particles, most of which are measured separately for all, red and blue galaxy populations. We find that our neighborhood abundance matching method accurately reproduces the central-subhalo relations, satellite fraction, the CLFs and CSMFs and the biases of galaxies. These features ensure that thus established galaxy-subhalo connections will be very useful in constraining galaxy formation processes. And we provide some suggestions on the three levels of using the galaxysubhalo pairs for galaxy formation constraints. The galaxy-subhalo links and the subhalo merger trees in the SDSS DR7 region extracted from our ELUCID simulation are available upon request.

Subject headings: dark matter - large-scale structure of the universe - galaxies: halos - methods: statistical
\end{abstract}

\section{INTRODUCTION}

To fully model the structure formation of the universe and to probe the detailed galaxy formation processes, one needs to have a fair sampling of the universe with sufficient large volume and resolution. Thanks to large redshift surveys, such as the Sloan Digital Sky Survey (SDSS; York et al. 2000), we are now able to carry out such kind of investigations to an unprecedented accuracy. However, in order to make full use of the potential of the observational data, one still has to develop or make use of optimal strategies. One of the most efficient ways of

\footnotetext{
${ }^{1}$ Department of Astronomy, Shanghai Key Laboratory for Particle Physics and Cosmology, Shanghai Jiao Tong University, Shanghai 200240, China; E-mail: xyang@sjtu.edu.cn

2 IFSA Collaborative Innovation Center, and Tsung-Dao Lee Institute, Shanghai Jiao Tong University, Shanghai 200240 China

${ }^{3}$ Shanghai Astronomical Observatory, Nandan Road 80 Shanghai 200030, China

${ }^{4}$ Key Laboratory for Research in Galaxies and Cosmology, Department of Astronomy, University of Science and Technology of China, Hefei, Anhui 230026, China

5 School of Astronomy and Space Science, University of Science and Technology of China, Hefei 230026, China

${ }^{6}$ Zhiyuan College, Shanghai Jiao Tong University, Shanghai 200240, China

${ }^{7}$ Department of Astronomy, University of Massachusetts, Amherst MA 01003-9305, USA

8 Astronomy Department and Center for Astrophysics, Tsinghua University, Beijing 10084, China

${ }^{9}$ Astronomy Department, Yale University, P.O. Box 208101, New Haven, CT 06520-8101

10 Purple Mountain Observatory, the Partner Group of MPI fr Astronomie, 2 West Beijing Road, Nanjing 210008, China

${ }^{11}$ Departamento de Física Teórica, Módulo 15, Facultad de Ciencias, Universidad Autónoma de Madrid, E-28049 Madrid, Spain
}

using the observational data is to carry out constrained simulations where the initial density field is indeed extracted from the observations, which is the basic idea of our ELUCID project.

Along this line, numerous attempts have been made to develop methods to reconstruct the initial conditions of structure formation in the local universe using galaxy distributions and/or peculiar velocities (Sousa et al. 2007; Jasche \& Wandelt 2013; Jasche et al. 2015; Sousa et al. 2015; Seliak et al. 2017). Hoffman \& Ribak (1991) developed a method to construct Gaussian random fields that are subjected to various constraints (see also Bertschinger 1987; van de Wevgaert \& Bertschinger 1996; Klypin et al. 2003; Kitaura \& Enßlin 2008). Klypin et al. (2003) improved this method by using Wiener Filter (see e.g. Zaroubi et al. 1995) to deal with sparse and noisy data. Gaussian density fields constrained by the peculiar velocities of galaxies in the local universe have also been used to set up the initial conditions for constrained simulations (e.g. Kravtsov, Klvpin, \& Hoffman 2002; Klypin et al. 2003; Gottloeber, Hoffman, \& Yepes 2010). Note, however, that the basic underlying assumption in this method is that the linear theory is valid on all scales (Klypin et al. 2003; Doumler et al. 2013).

In a recent paper, Wang et al. (2014, hereafter ELUCID I) developed a method combining the Bayesian reconstruction approach with a much more accurate dynamic model of structure evolution, the Particle Mesh (PM) model. The PM technique has been commonly adopted in $N$-body codes to evaluate gravitational forces on relatively large scales (see e.g. White. Frenk, \& Davis 1983; Klypin \& Shandarin 1983; 
Jing \& Suto 2002; Springel 2005), and can follow the structure evolution accurately as long as the grid cells and time steps are chosen sufficiently small. Tests show that this method can achieve much higher reconstruction accuracy than any other methods in the literature. To apply this method to observation, one needs to reconstruct the cosmic density field of the local Universe. As illustrated in Wang et al. (2009), this density field can be fairly well reconstructed using the distribution of (relatively massive) galaxy groups (i.e., dark matter halos). Using the galaxy groups (Yang et al. 2007, 2012) extracted from the SDSS Data Release 7 (Abazajian et al. 2009, DR7), Wang et al. (2012) have obtained the mass, tensor and velocity fields of the local universe in the SDSS DR7 region.

With all these preparations, in a recent study, Wang et al. (2016, hereafter ELUCID III), make use of the density field re-constructed from the north galactic pole of SDSS DR7 region with an improved domain mass assign method (Wang et al. 2013) to predict the evolution of structure of the local universe enclosed in a $500 h^{-1} \mathrm{Mpc}$ length cubic box. As shown in ELUCID I, the reconstruction can recover more than half of the phase information down to a scale $k \sim 3.4 \mathrm{hMpc}^{-1}$ at $z=0$. Tests using original and reconstructed simulations show that more than half of the halos with mass $\gtrsim 10^{13.5} h^{-1} \mathrm{M}_{\odot}$ can be reliably reproduced in which more than $50 \%$ particles are in common with the counterpart halos in the original simulation (see Tweed et al. 2017, hereafter ELUCID II). These features indicate that the halos formed in the SDSS DR7 region in our ELUCID simulation have roughly consistent large scale environment as the true universe and the evolution of massive halos can be roughly well modelled.

In this paper, we propose a novel neighborhood abundance matching method to link galaxies observed in the SDSS DR7 region with halos/subhalos in our ELUCID simulation in the local same small volumes. Once the galaxy-subhalo connections are generated, we can use them to constrain semi-analytical galaxy formation models (SAMs) in an halo based and local environment based apple-to-apple comparisons. Note that, technically, we can make the neighborhood abundance matching between galaxy groups and dark matter halos as well. However, because of the following a few reasons, we decide to use galaxies rather than groups. The main reason is that since some massive groups in the observation may split (or connected) with respect to halos in the simulation, then a group-halo matching may over predict the galaxy population in one halo and under predict it in the other. The second reason is that since only massive groups can be well reproduced in the ELUCID simulation, we are not able to use the group-halo connections in individual low mass halos. In addition, it would be interesting to see the impact of interlopers in the galaxy groups which is not available in the group-halo matching (see Campbell et al. 2015, for the related discussions).

The structure of this paper is organized as follows. Section 2 gives a detailed description of the data we used in this study, including the halos/subhalos extracted from the ELUCID simulations, the SDSS DR7 galaxy catalog, as well as the neighborhood abundance matching between galaxies and dark matter subhalos. In Section 3 we probe the central-subhalo relations, satellite fraction, CLFs and CSMFs of galaxies. In Section 4 we measure the cross correlation functions between galaxies and the dark matter particles. In Section 5 we provide some suggestions for the usage of the galaxy-subhalo connections established in this work. Finally, we summarize our results in Section 6. Throughout the paper we adopt a $\Lambda \mathrm{CDM}$ cosmology with parameters that are consistent with the fifth-year data release of the WMAP mission (hereafter WMAP5 cosmology): $\Omega_{\mathrm{m}}=0.258, \Omega_{\Lambda}=$ $0.742, \Omega_{\mathrm{b}}=0.044, h=H_{0} /\left(100 \mathrm{~km} \mathrm{~s}^{-1} \mathrm{Mpc}^{-1}\right)=0.72$ and $\sigma_{8}=0.80$ (Dunkley et al. 2009).

\section{DATA}

\subsection{The halos/subhalos in the ELUCID simulation}

In this study we use dark matter halos/subhalos extracted from the ELUCID simulation. This simulation which evolves the distribution of $3072^{3}$ dark matter particles in a periodic box of $500 h^{-1} \mathrm{Mpc}$ on a side was carried out in the Center for High Performance Computing, Shanghai Jiao Tong University. The simulation was run with L-GADGET, a memory-optimized version of GADGET2 (Springel 2005). The cosmological parameters adopted by this simulation are consistent with WMAP 5 results with each particle has a mass of $3.0875 \times 10^{8} h^{-1} \mathrm{M}_{\odot}$. In our ELUCID simulation, we make use of the mass density field extracted from the galaxy/group distribution in the north galactic pole region of the SDSS DR7 to constrain the initial conditions using a Hamiltonian Markov Chain Monte Carlo method (HMCMC) with particle mesh dynamics (see ELUCID I \& III for details). As an illustration, we show in Fig. 1 the distributions of the related galaxies, reconstructed and simulated mass density fields. Shown in the left hand panel is a slice of galaxy distributions in the north galactic pole of SDSS DR7. In the middle panel is a slice of density field constructed from these galaxy distributions. In the right panel is the mass density field revealed (evolved to redshift $z=0$ ) in our ELUCID simulation. The enclosed region in the left panel with solid lines is the density field supposed to be reproduced in our ELUCID simulation. The basic properties of our ELUCID simulation, including the algorithm to perform the simulation, as well as the output power spectrum, halo mass functions, etc. can be found in ELUCID III.

From the ELUCID simulation, dark matter halos were first identified by a friends-of-friends (FOF) algorithm with linking length of 0.2 times the mean particle separation and containing at least 20 particles. The dark matter halo mass function of this simulation at redshift $z=0$ is checked and agrees very well with the model predictions of Sheth. Mo, \& Tormen (2001) and Tinker et al. (2008). Based on halos at different outputs, we first use the SUNFIND algorithm (Springel et al. 2001) to identify the bound substructures in the FOF halos. The most massive substructure in a FOF halo is considered as the main halo of this FOF and all the other subhalos in this FOF are called subhalos. For a given subhalo or main halo, each particle is assigned a weight which decreases with the binding energy. We then find all main halos and subhalos in the subsequent snapshot that contain some of its particles. We count these particles with weight for these potential descendants. The candidate with highest 

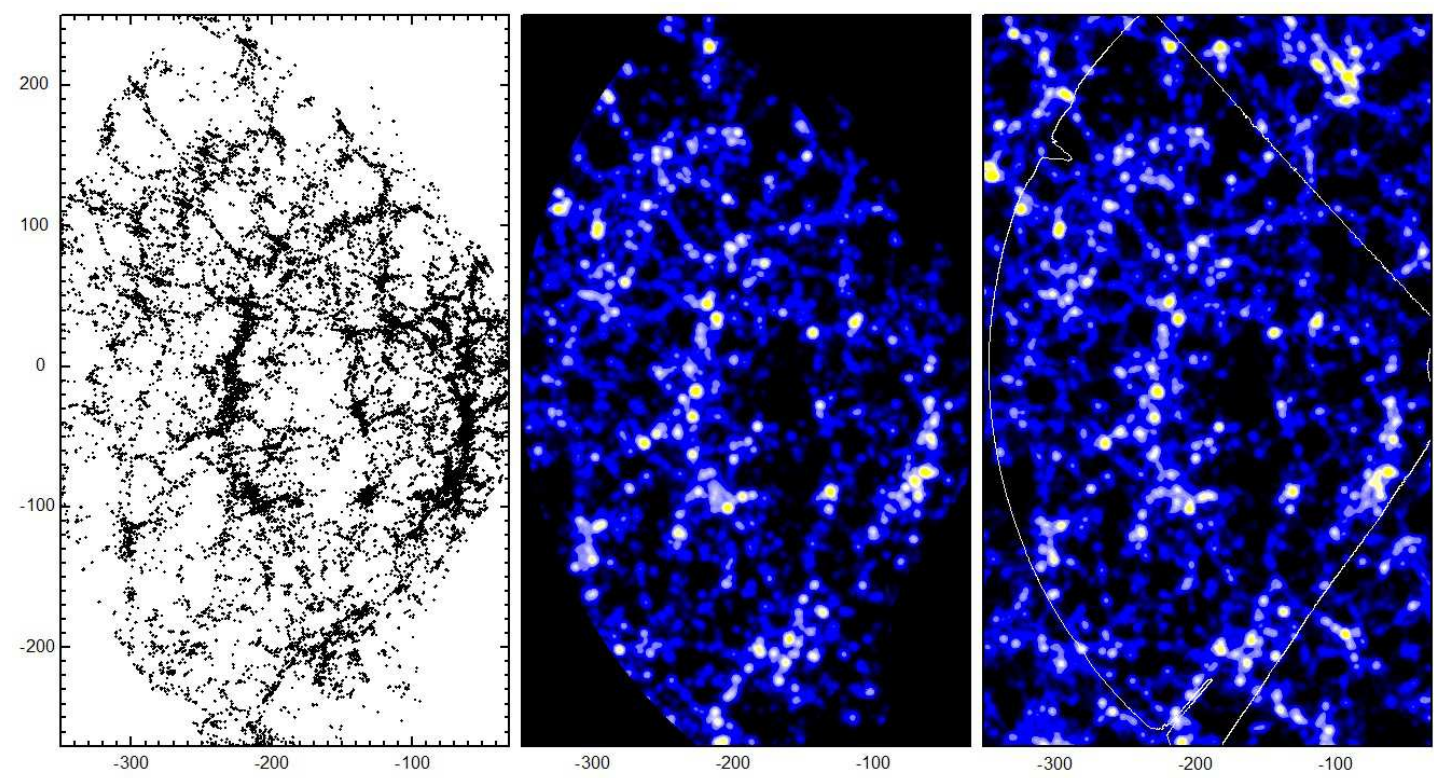

FIG. 1. - The sketch of the SDSS DR7 galaxy and density fields in the ELUCID simulation. Shown in the left hand panel is a slice of galaxy distributions in the north galactic pole of SDSS DR7. In the middle panel is a slice of density field constructed from these galaxy distributions. In the right panel is the mass density field revealed in our ELUCID simulation. The enclosed region with solid lines is the density field supposed to be reproduced in the ELUCID simulation.

weighted count is selected as the descendant. Please see Springel et al. (2005) for the details.

In order to properly link galaxies with dark matter halos and subhalos, especially those satellite galaxies, one needs to properly treat the subhalos in the simulations (see Jiang \& van den Bosch 2016, for the related discussions). A widely adopted subhalo population in SHAMs are the mass or circular velocity of survived subhalos extracted by sub-finders in the simulation, but their masses/velocities are updated to the maximum values along their accretion histories (e.g. Conroy et al. 2006; Hearin et al. 2013). This method implies that: (1) each subhalo can form only one galaxy, (2) the central-host halo relation does not evolve significantly with redshift, and (3) satellite galaxies disrupt whenever subhalos can no longer be identified in the simulation, either because of limiting mass resolution or because the subhalo is tidally disrupted. An alternative method in the SHAM is to separate the central and satellite galaxies, i.e., one can make abundance matching separately for central galaxies v.s. main halos and satellite galaxies v.s. subhalos (see Rodríguez-Puebla et al. 2015, for a similar attempt). In this regard, the evolution of satellite galaxy will be automatically taken into account. In this study, we will perform both of these two kinds of abundance matching methods and compare the differences between them. We refer the former as 'Match1' and the later as 'Match2'.

In order to match the galaxies in the SDSS observations, we first rotate the simulation box so that the resimulated density field is in superposition with the SDSS observation region. We then discard the dark matter subhalos that are outside the survey sky coverage region used for our density field re-construction. The $r a$, dec and $z_{\text {com }}$ of the subhalos are calculated from their real space positions. Then their final redshifts are obtained by adding the peculiar velocities, with $z_{\mathrm{obs}}=z_{\mathrm{com}}+v_{\text {pec }} / c$. We trim subhalos within the redshift range $0<z<0.12$ for our subsequent matching with galaxies in observations.

\section{2. $S D S S D R 7$}

The galaxy catalog we used for finding galaxy groups, making density re-construction and performing the ELUCID simulation is the New York University Value-Added Galaxy Catalog (NYU-VAGC; Blanton et al. 2005). The catalog is compiled based on SDSS DR7 (Abazajian et al. 2009) but with an independent set of significantly improved reductions. From the NYU-VAGC, we select all galaxies in the Main Galaxy Sample with an extinction corrected apparent magnitude brighter than $r=17.72$, with redshifts in the range $0.01 \leq z \leq 0.20$ and with a redshift completeness $\mathcal{C}_{z}>0.7$. This gives a sample of 639,359 galaxies with a sky coverage of 7748 square degrees. In this study, we make use of all the galaxies in this sample for our investigation. Within these 639,359 galaxies, 35, 678 do not have spectroscopic redshifts, and are assigned with redshifts from their nearest neighborhoods.

According to Yang et al. (2007, hereafter Y07), the absolute magnitudes of galaxies in bandpass $Q$ are computed using

$$
{ }^{0.1} \mathrm{M}_{Q}-5 \log h=m_{Q}+\Delta m_{Q}-\mathrm{DM}(z)-K_{Q}-E_{Q} .
$$

Here $\operatorname{DM}(z)=5 \log \left[D_{L} /\left(h^{-1} \mathrm{Mpc}\right)\right]+25$ is the bolometric distance modulus calculated from the luminosity distance $D_{L}$ using a WMAP5 cosmology. $\Delta m_{Q}$ is the latest zero-point correction for the apparent magnitudes, which converts the SDSS magnitudes to the $\mathrm{AB}$ system, and for which we adopt $\Delta m_{Q}=(-0.036,+0.012,+0.010,+0.028,+0.040)$ for $Q=(u, g, r, i, z)$. All absolute magnitudes are $K+E$ corrected to $z=0.1$. For the $K$ corrections we use the latest version of 'Kcorrect' ( $\mathrm{v} 4$ ) described in Blanton et al. (2003; see also Blanton \& Roweis 2007), 
Yang et al.

which we apply to all galaxies that have meaningful magnitudes and meaningful redshifts, including those that have redshifts from alternative sources and those that have been assigned the redshift of their nearest neighbor. Finally, the evolution corrections to $z=0.1$ are computed using $E_{Q}=A_{Q}(z-0.1)$, with $A_{Q}=$ $(-4.22,-2.04,-1.62,-1.61,-0.76)$ for $Q=(u, g, r, i, z)$ (see Blanton et al. 2003). Note that these evolution corrections imply that galaxies were brighter in the past (at higher redshifts).

In addition to the absolute magnitudes, we also compute for each galaxy its stellar mass, $M_{*}$. Using the relation between stellar mass-to-light ratio and color of Bell et al. (2003), we obtain

$$
\begin{aligned}
\log \left[\frac{M_{*}}{h^{-2} \mathrm{M}_{\odot}}\right]= & -0.306+1.097\left[{ }^{0.0}(g-r)\right]-0.1 \\
& -0.4\left({ }^{0.0} \mathrm{M}_{r}-5 \log h-4.64\right) .
\end{aligned}
$$

Here ${ }^{0.0}(g-r)$ and ${ }^{0.0} \mathrm{M}_{r}-5 \log h$ are the $(g-r)$ color and $r$-band magnitude $K+E$ corrected to $z=0.0,4.64$ is the $r$-band magnitude of the Sun in the AB system (Blanton \& Roweis 2007), and the -0.10 term effectively implies that we adopt a Kroupa (2001) IMF (Borch et al. 2006). For a small fraction (about 2\%) of all galaxies, the $g-r$ color that results from the photometric SDSS pipeline is unreliable. These galaxies typically have $g-r$ colors that are clearly unrealistic (they are catastrophic outliers in the color-magnitude distribution). If this is not accounted for, equation (2) assigns these galaxies stellar masses that are unrealistically high or low. To take account of these outliers we follow Y07 using the color bi-Gaussian distributions of galaxies obtained in Yang et al. (2008; see also Li et al. 2006). For any galaxy that falls outside the 3 - $\sigma$ ranges from the mean color-magnitude relations of both the red sequence and the blue cloud (about $2 \%$ of all galaxies), we compute its stellar mass using the mean color of the red sequence (when the galaxy is too red) or the blue cloud (when the galaxy is too blue).

In order to probe the color dependence of galaxies, following Yang et al. (2008), we separate our galaxies with ${ }^{0.1}(g-r)<0.9$ into red and blue subsamples using the criteria,

$$
{ }^{0.1}(g-r)=1.022-0.0651 x-0.00311 x^{2},
$$

where $x={ }^{0.1} \mathrm{M}_{r}-5 \log h+23.0$. While galaxies with ${ }^{0.1}(g-r) \geq 0.9$ are directly attributed to red subsample.

From the above galaxy catalog, we only select galaxies within groups that were used to map the density field and thus the initial density field in our ELUCID simulation. That is we only select galaxies which are located within the range: $99<r a<283,-7<$ dec $<75$ (i.e. in the north galatic pole) and redshift $0.01<z<0.12$. After this selection, a total of 396069 galaxies are remained for our subsequent probes.

\subsection{The neighborhood abundance matching method}

In order to link galaxies with dark matter (sub)halos, one can either establish the HOD or CLF models (e.g. Jing et al. 1998; Peacock \& Smith 2000; Yang et al. 2003; van den Bosch et al. 2003; Zheng et al. 2005; Tinker et al. 2005; Cooray
2006; van den Bosch et al. 2007; Brown et al. 2008; More et al. 2009; Cacciato et al. 2009; Neistein et al. 2011; Avila-Reese \& Firmani 2011; Leauthaud et al. 2011; Yang et al. 2012; Rodríguez-Puebla et al. 2015; Li et al.|2016; Zu \& Mandelbaum 2016; Bull|2017; Cohn 2017; Contreras et al. 2017; Rodríguez-Puebla et al. 2017), or via subhalo abundance matching processes (e.g. Vale \& Ostriker 2004; Vale \& Ostriker 2006; Conrov et al. 2006; Shankar et al. 2006; Conroy et al. 2009; Moster et al. 2010; Guo et al. 2010; Behroozi et al. 2010; Hearin et al. 2013). These probes have revealed many observational features of galaxies, and were widely used to constrain the galaxies formation models. In these studies, however, only the global properties of galaxies such as the stellar mass functions/luminosity functions, and clusterings are used to establish the galaxy-(sub)halo connections. In this probe, as the structures in our ELUCID simulation are supposed to trace the evolution of real structures in our SDSS DR7 region in consideration, we set out to match galaxies with the dark matter subhalos in their neighborhood, i.e., using a neighborhood abundance matching method.

We first sort the stellar masses of the galaxies. Starting from the most massive galaxy, we search in redshift space for each subhalo sample the most likely subhalo in a small volume in its neighborhood, which is then marked as its counterpart. The likelihood of the subhalo to be linked with the candidate galaxy is modelled as follows,

$$
P\left(r_{p}, \pi, M_{s h}\right)=M_{s h} \exp \left(-\frac{r_{p}^{2}}{2 r_{\text {off }}^{2}}\right) \exp \left(-\frac{\pi^{2}}{2 v_{\text {off }}^{2}}\right) .
$$

Here $r_{p}$ and $\pi$ are the separations between the galaxy and subhalo in the perpendicular and along the line of sight directions, respectively. $M_{h}$ is the mass of subhalo in consideration. While $r_{\text {off }}$ and $v_{\text {off }}$ are the two free parameters we choose to make our neighborhood abundance matching. In the extreme case where $r_{\text {off }}=\infty$ and $v_{\text {off }}=$ $\infty$, the neighborhood abundance matching method degrades to the traditional abundance matching method. We use two sets of parameters to perform our neighborhood abundance matching: (1) $r_{\text {off }}=2.5 h^{-1} \mathrm{Mpc}$ and $v_{\text {off }}=500 \mathrm{kms}^{-1}$ and $(2) r_{\text {off }}=5.0 h^{-1} \mathrm{Mpc}$ and $v_{\text {off }}=1000 \mathrm{kms}^{-1}$, and compare their performances. Note that in our ELUCID simulation, the reconstructed density field is smoothed using a Gaussian kernel of radius $2.0 h^{-1} \mathrm{Mpc}$. Here, these two sets of choices are made according to a compromise of the scatters and the separations between the galaxies and subhalos, which will be illustrated as follows. Using these criteria, we sequentially search for all the galaxies their counterparts in redshift space within a maximum distance $\leq 30 h^{-1} \mathrm{Mpc}$. For the total of 396069 galaxies, according to criteria (2), there are 296488 central galaxies that are linked with the main halos and 99581 satellite galaxies that are linked with the subhalos for Match1 method. Criteria (1) gives very similar numbers, with typical differences at a few hundreds. Comparing to the ones specified in the group catalog, 277139 centrals and 118930 satellites, the Match1 method roughly underestimated about $\sim 20 \%$ satellite galaxy population. On the other hand, by definition, the Match2 method will give the same central/satellite separation as those in groups. 

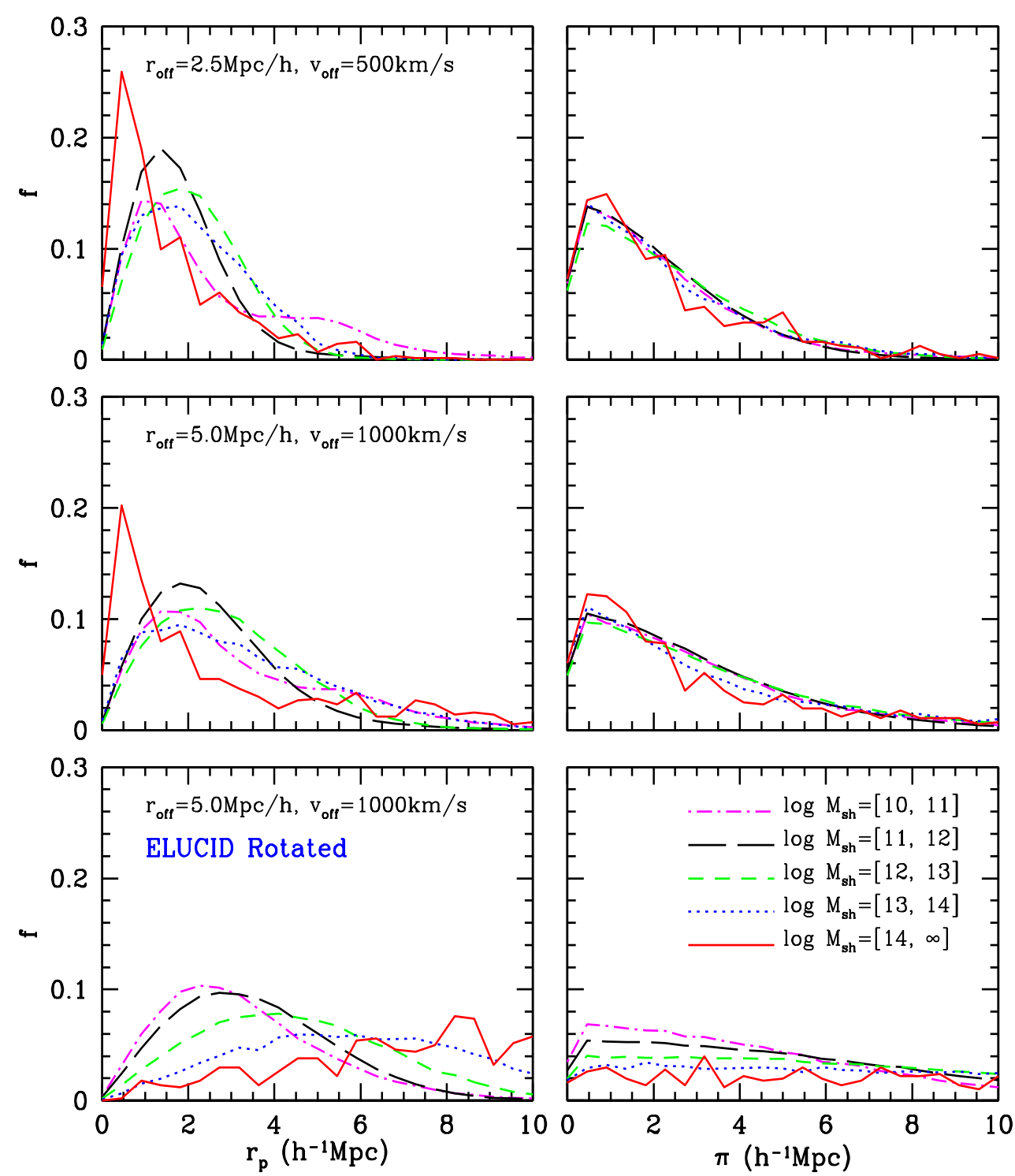

Fig. 2.- The separation distributions of galaxy-subhalo pairs in different subhalo mass bins using different style lines as indicated. Here we only show results obtained from Match1 method. Shown in the left and right panels are the $r_{p}$ and $\pi$ distributions, respectively. Results shown in the upper panels are obtained using criteria (1): $r_{\text {off }}=2.5 h^{-1} \mathrm{Mpc}$ and $v_{\text {off }}=500 \mathrm{~km} \mathrm{~s}^{-1}$. Results shown in the middle panels are obtained using criteria (2): $r_{\text {off }}=5.0 h^{-1} \mathrm{Mpc}$ and $v_{\text {off }}=1000 \mathrm{~km} \mathrm{~s}^{-1}$. While the results shown in the lower panels are obtained from a rotated (i.e. unmatched) version of the ELUCID simulation for matching criteria (2).

We show in Fig. 2 the separation distributions of galaxy-subhalo pairs in different subhalo mass bins. Shown in the upper-left panel are the $r_{p}$ distributions for matching criteria (1): $r_{\text {off }}=2.5 h^{-1} \mathrm{Mpc}$ and $v_{\text {off }}=$ $500 \mathrm{~km} \mathrm{~s}^{-1}$. Note that as the results for Match1 and Match2 methods are very similar, here we only show those obtained from Match1 method. As we can see, the offset between galaxies and subhalos in the most massive mass bin with $\log M_{h} \geq 14.0$, which peaks at $\sim$ $0.5 h^{-1} \mathrm{Mpc}$, is the smallest. About $50 \%$ of the matched pairs have projected separations less than $2 h^{-1} \mathrm{Mpc}$. The lower mass subhalos have slightly larger separations and the distributions peak at about $1.5-2.0 h^{-1} \mathrm{Mpc}$. About $50 \%$ of the matched pairs have projected separations less than $2.5 h^{-1} \mathrm{Mpc}$. Shown in the upper-right panel are the $\pi$ distributions of galaxy-subhalo pairs for match- ing criteria (1). The offsets for subhalos in different mass bins are quite similar. All the distributions peak at $\sim 50 \mathrm{~km} \mathrm{~s}^{-1}$ and about $50 \%$ of the matched pairs have line of sight separation less than $200 \mathrm{~km} \mathrm{~s}^{-1}$.

The results shown in the middle panels of Fig. 2 are similar to those shown in the upper panels, but for matching criteria (2): $r_{\text {off }}=5.0 h^{-1} \mathrm{Mpc}$ and $v_{\text {off }}=$ $1000 \mathrm{~km} \mathrm{~s}^{-1}$. The overall distribution properties are quite similar to those of matching criteria (1), but with slightly larger offsets. That is, about $50 \%$ of the matched pairs have projected separations less than $3.0 h^{-1} \mathrm{Mpc}$ and line of sight separation less than $250 \mathrm{~km} \mathrm{~s}^{-1}$.

As we have matched galaxies with subhalos, it is quite straightforward to check their luminosity (stellar mass) - subhalo mass relations. Show in the upper panels of Fig. 3 are the luminosity - subhalo mass (left panel) and 

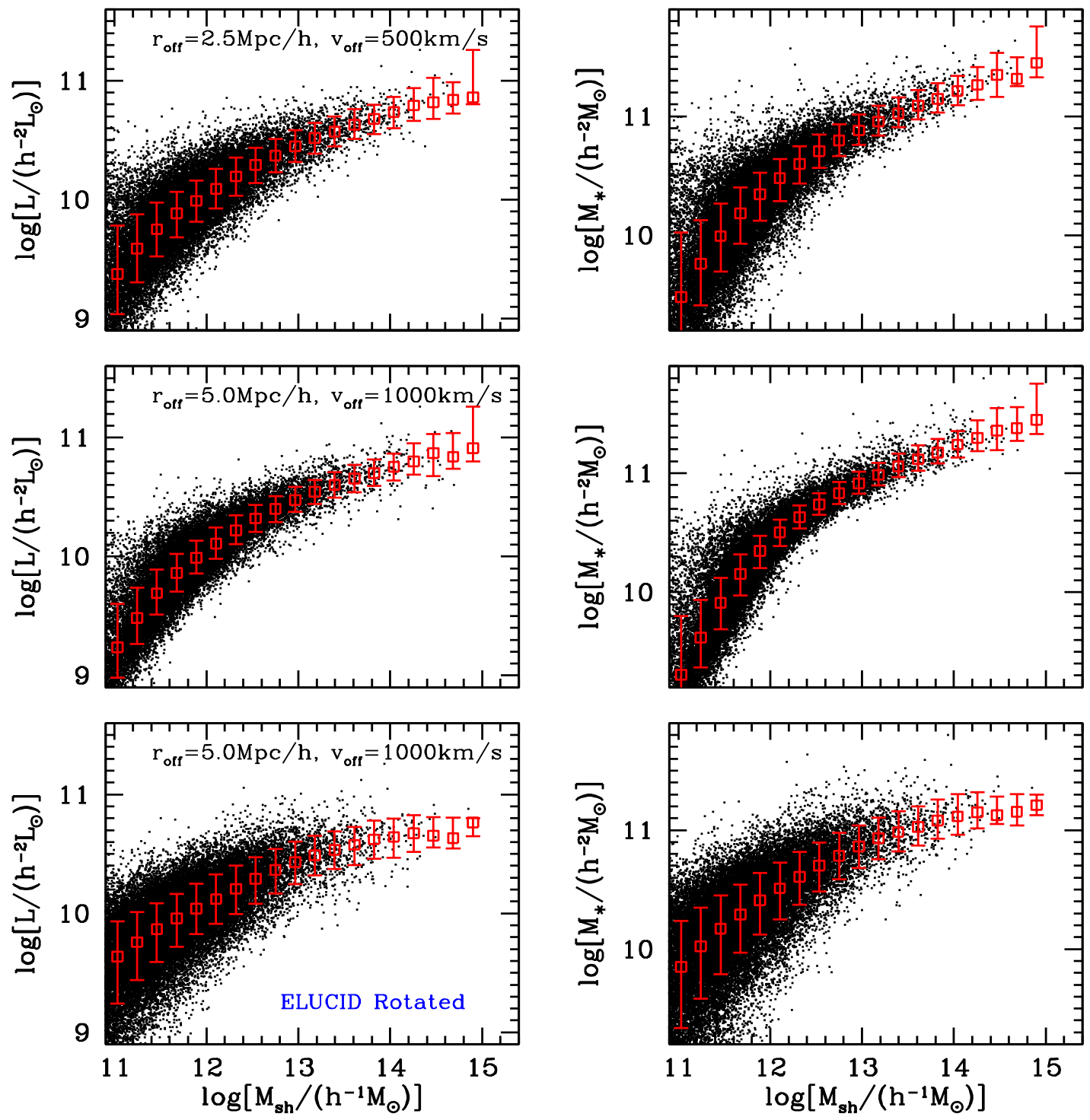

FIG. 3. - The luminosity (left panels) and stellar mass (right panels) v.s. the subhalo mass $\log M_{s h}$ in the ELUCID simulation of the matched galaxy-subhalo pairs. Shown in the upper and middle panels are results obtained from Match1 method for matching criteria (1) and (2), respectively. While the results shown in the lower panels are obtained from a rotated (i.e. unmatched) version of the ELUCID simulation for matching criteria (2).

stellar mass - subhalo mass (right panel) relations for our matching criteria (1). Shown in the middle panels are results for our matching criteria (2). Here again, we only show results obtained from Match1 method. While results obtained from Match2 are very similar. In each panel, the open squares with error-bars indicate the median and $68 \%$ confidence levels of these relations of all the galaxies. Comparing the results for the two matching criteria, the latter shows somewhat tighter luminosity (stellar mass) - subhalo mass relations, especially in the low mass subhalo. We thus think the latter matching criteria works better. In what follows, we only present results obtained using the matching criteria (2), i.e., with $r_{\text {off }}=5.0 h^{-1} \mathrm{Mpc}$ and $v_{\text {off }}=1000 \mathrm{~km} \mathrm{~s}^{-1}$.

Before we proceed to provide more detailed tests on the performance of our neighborhood abundance matching method on the ELUCID simulation, it would be in- teresting to check the above separation distribution and luminosity (stellar mass) - subhalo mass relations if the neighborhood abundance matching method is applied to a simulation that does not have good correspondence with the observation. For this purpose, we rotate the ELUCID simulation box by 90 degree and shift it by $250 h^{-1} \mathrm{Mpc}$, and then perform the same procedures using matching criteria (2). Note that after such a treatment, the simulation density field is no longer matched with the SDSS density field. Shown in the lower panels of Figs. 2 and 3 are the resulting separation distributions and luminosity (stellar mass) - subhalo mass relations. We can see that the separation distributions of galaxy-subhalo pairs in this situation are very different from our fiducial case, especially for massive clusters. The very large separation between galaxies and massive (sub)halos indicates that the galaxy-subhalo pairs might 

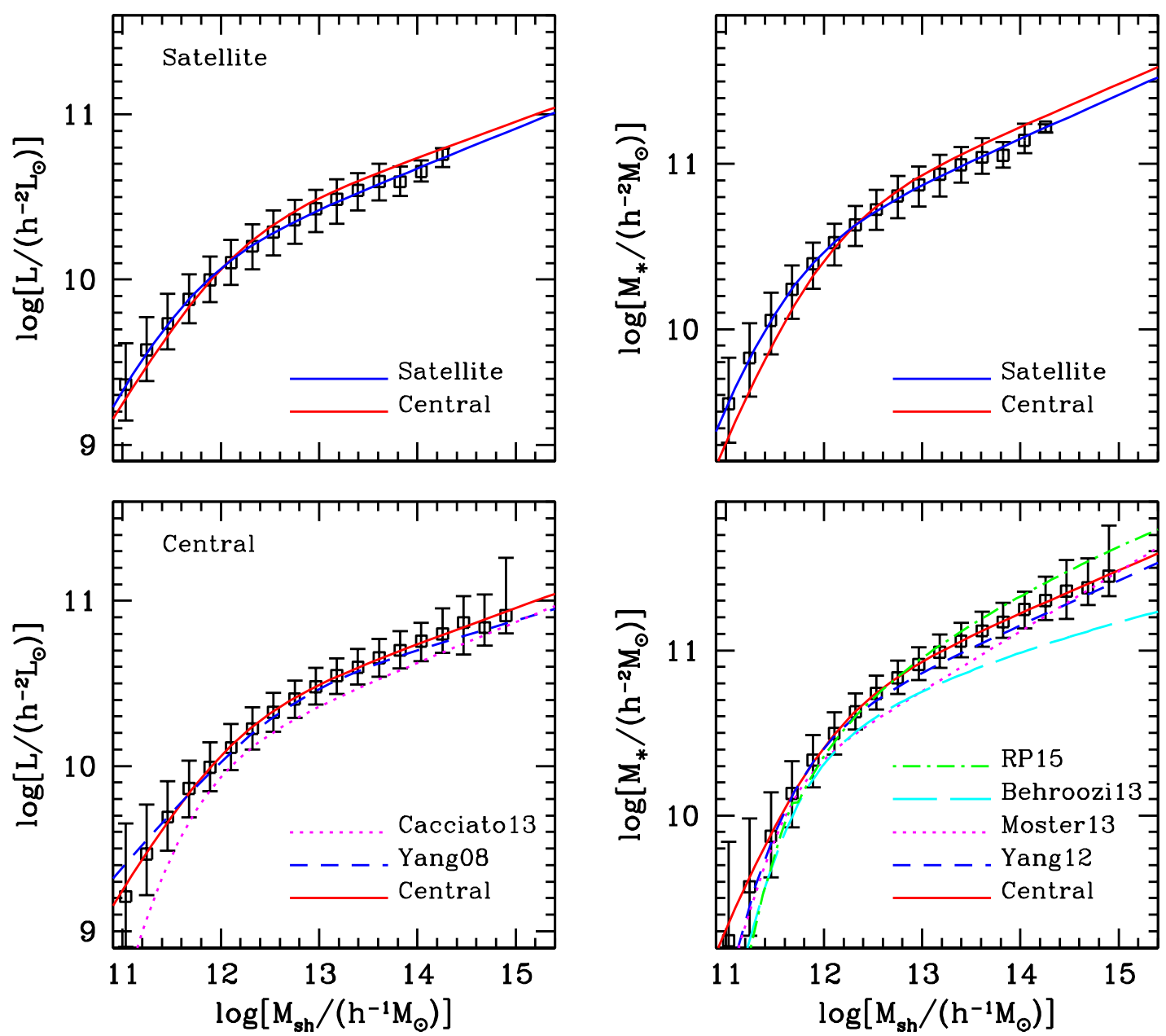

Fig. 4. - The luminosity (left panels) and stellar mass (right panels) v.s. the subhalo mass $\log M_{h}$ in the ELUCID simulation of the matched galaxy-subhalo pairs. Shown in the upper and lower panels are results obtained from Match2 method for central and satellite galaxies, respectively. The red and blue solid lines in these panels are the best fitting results for central and satellite galaxies separately. For comparison, the blue dashed and magenta dotted dashed lines in the left panel are results obtained by Yang et al. (2008) and Cacciato et al. (2013), respectively. The blue dashed, magenta dotted, cyan long dashed and green dot-dashed lines are results obtained by Yang et al. (2012), Moster et al. (2013), Behroozi et al. (2013) and Rodríguez-Puebla et al. (2015), respectively.

come from different origins. For small (sub)halos with mass $\lesssim 10^{12.0} h^{-1} \mathrm{M}_{\odot}$, the difference is quite small, indicating that the low mass galaxy-subhalo pairs, even in the ELUCID simulation might dominated by Poisson errors (see Tweed et al. 2017). In addition, the luminosity (stellar mass) - subhalo mass relations in this case are much worse, i.e., with much larger scatters, than those of our fiducial cases. In general, as we mentioned, if we set $r_{\mathrm{off}}=\infty$ and $v_{\mathrm{off}}=\infty$, the neighborhood abundance matching method will degrade to the traditional abundance matching method, which will provide monotonic luminosity (stellar mass) - subhalo mass relations.

\section{THE HALO OCCUPATION DISTRIBUTIONS OF GALAXIES}

After we matched galaxies with subhalos in the ELUCID simulation, we proceed to measure a few galaxy statistics within host halos of different masses. These statistics are compared to those obtained from galaxy groups (e.g. Yang et al. 2008, 2009) to demonstrate the feasibility of populating dark matter subhalos with ob- served galaxies via the neighborhood abundance matching method outlined in section 2.3 .

\subsection{The galaxy-subhalo luminosity/mass relations}

Here we start our probe using the data obtained from Match2 method. Shown in the upper and lower panels of Fig. 4 are the luminosity (stellar mass) - subhalo mass relations for satellite and central galaxies separately. We follow Yang et al. (2008) to use the following $L-M_{s h}$ functional form to describe the median luminosity - subhalo mass relation,

$$
L=L_{0} \frac{\left(M_{s h} / M_{1}\right)^{\alpha+\beta}}{\left(1+M_{s h} / M_{1}\right)^{\beta}} .
$$

This model contains four free parameters: a normalized luminosity, $L_{0}$, a characteristic halo mass, $M_{1}$, and two slopes, $\alpha$ and $\beta$. The blue and red solid lines shown in the left panels are the best fits to the average $L-M_{s h}$ relations for satellite and central galaxies, respectively. The best fitting parameters are listed in Table 1 in the first and second rows. Although not very significant, we 

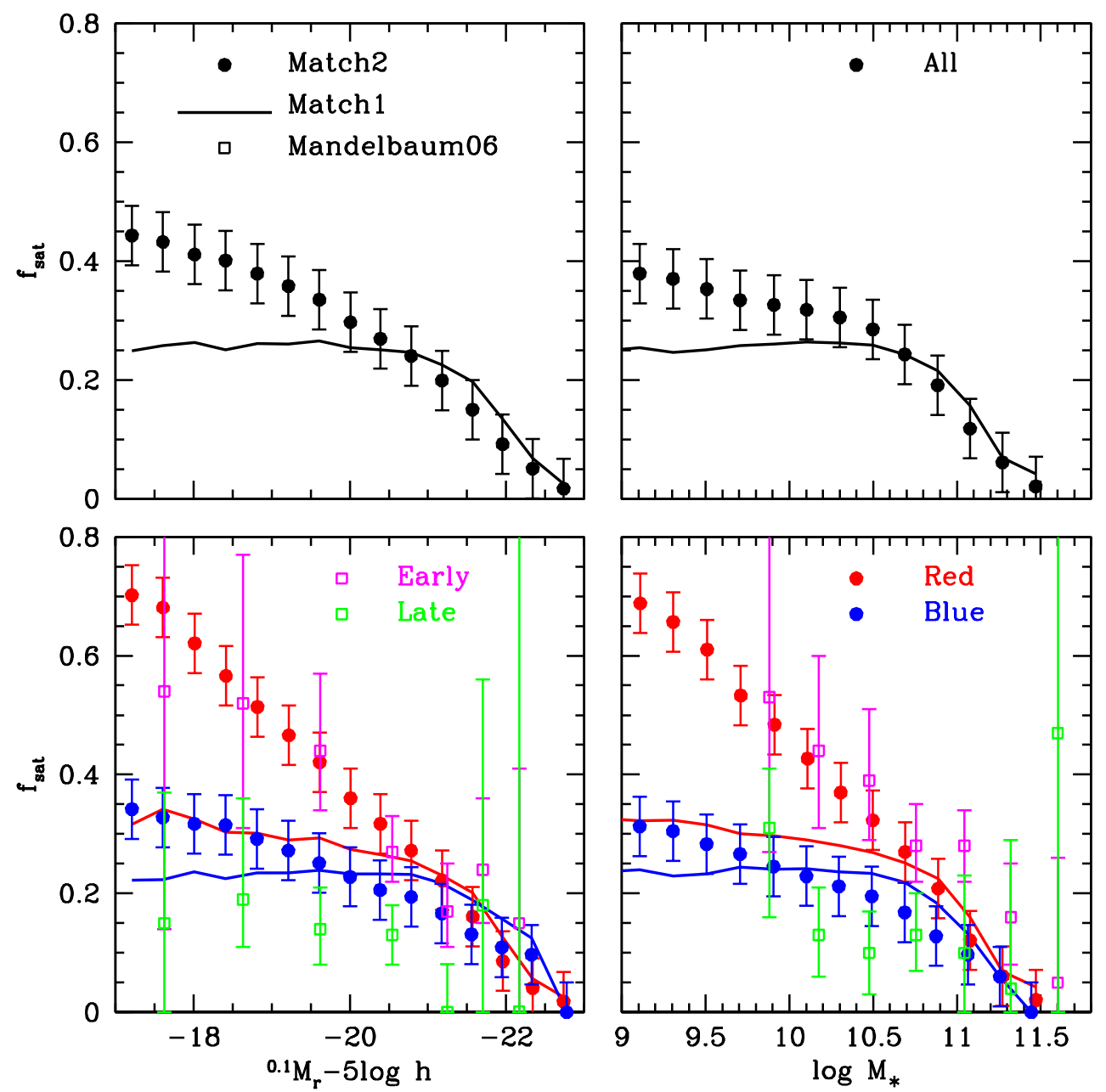

FiG. 5.- Fraction of satellite galaxies as function of luminosity (left panels) and stellar mass (right panels). Results are shown separately for all (upper panels), red and blue galaxies (lower panels), respectively. The solid dots with error bars represent the results obtained for Match2 method. Lines are results for Match1 method. The open squares with error bars are results obtained by Mandelbaum et al. (2006) for comparison.

do see some differences between the luminosity - subhalo mass relations of central and satellite galaxies, indicating that satellite galaxies may have different stripping or disruption effect from subhalos.

For comparison, we also show in the lower-left panel the best fitting results obtained by Yang et al. (2008) using a dashed line, where the set of best fitting parameters are listed in the third row of Table 1. This set of result is obtained from SDSS galaxy group catalog directly, in which (i) it is assumed that central galaxies are the brightest group members, and (ii) halo masses are inferred using abundance matching of host halos to the total stellar mass of the groups. The dotted line shown in that panel are the results obtained by Cacciato et al. (2013) by CLF model constraints using the clustering and weak lensing measurements of galaxies. Overall, our neighborhood abundance matching method gives quite consistent $L-M_{s h}$ relation with these previous measurements, except that of Cacciato et al. (2013). The slight systematic deviation from Cacciato et al. (2013) is mainly caused by the different definition of halo mass and the cosmology they used. As shown in Fig. 7 of Cacciato et al. (2013), if the halo mass definition and cos- mology are properly converted, their results agree with those obtained by Yang et al. (2008) very well.

For the $M_{*}-M_{s h}$ relations shown in the right panels, we use a similar function to fit the data:

$$
M_{*}=M_{0} \frac{\left(M_{s h} / M_{1}\right)^{\alpha+\beta}}{\left(1+M_{s h} / M_{1}\right)^{\beta}} .
$$

The blue and red solid lines shown in the right panels are the best fits of this model to the data for the satellite and central galaxies separately, where the best-fit parameters are listed in the fourth and fifth rows of Table 1.

For comparison, we also show in the lower-right panel, the model constraints obtained by Yang et al. (2012) using a dashed line, where the related set of parameters are listed in the sixth row of Table 1 . The model constraints obtained by Moster et al. (2013), Behroozi et al. (2013) and Rodríguez-Puebla et al. (2015) are shown as the dotted, long dashed and dot-dashed lines, respectively. Here again, we see our neighborhood abundance matching method gives quite consistent $M_{*}-M_{s h}$ relation with these previous probes, except that of Behroozi et al. (2013), which is somewhat lower especially at massive 

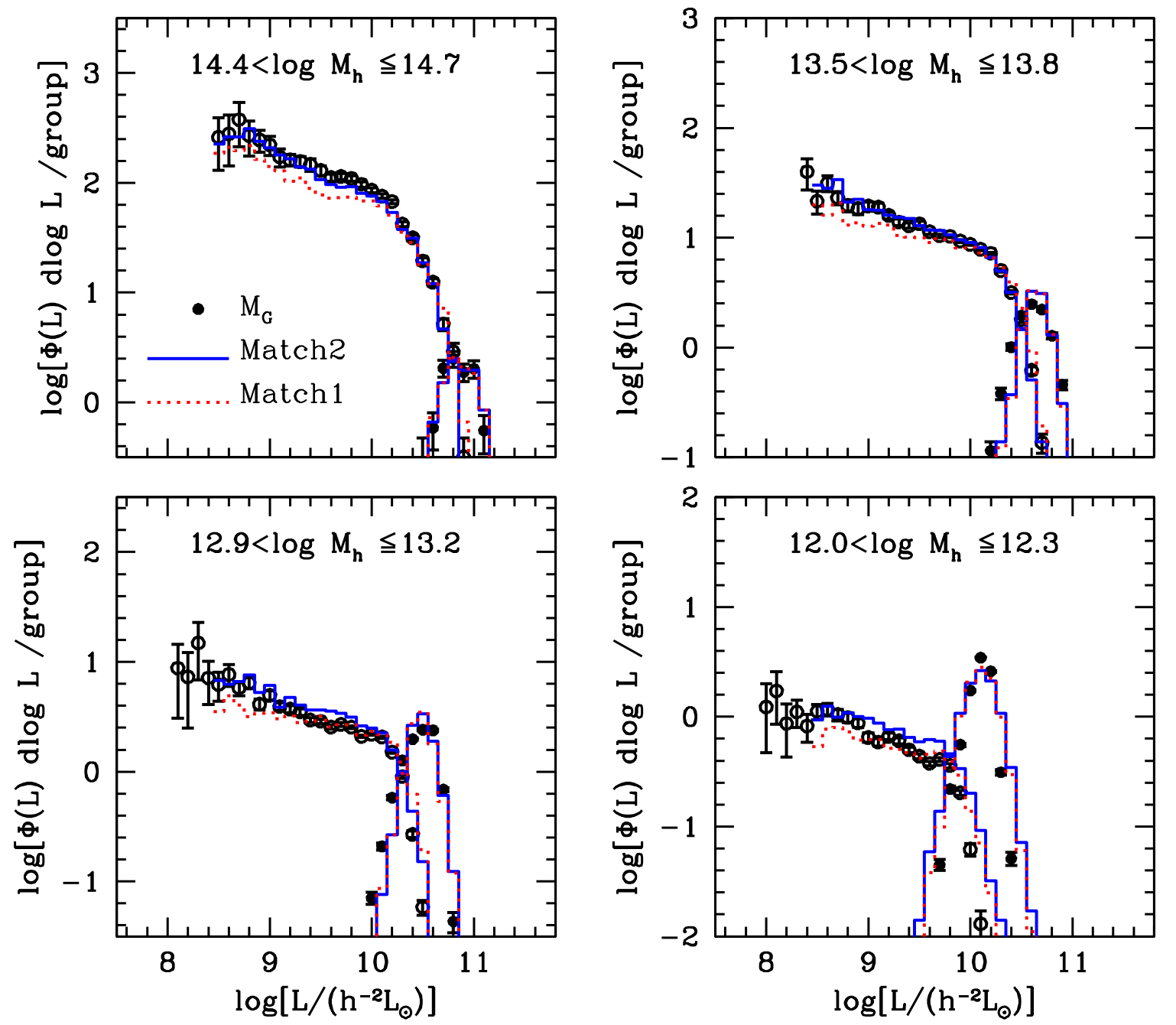

FIG. 6. - The conditional luminosity functions (CLFs) of galaxies in groups and host halos of different masses. Symbols correspond to the CLFs obtained from SDSS galaxy groups, with solid and open circles indicating the contributions from central and satellite galaxies, respectively. The errorbars reflect the 1- $\sigma$ scatter obtained from 200 bootstrap samples. The solid and dotted lines are results obtained for the Match2 and Match1 methods, respectively.

end. The difference is mainly caused by adopting a different stellar mass estimation method (see Behroozi et al. 2013, for a similar trend and the related discussions).

TABLE 1

THE BEST FITTING PARAMETERS.

\begin{tabular}{lcccc}
\hline \hline Sample & $\log L_{0}$ & $\log M_{1}$ & $\alpha$ & $\beta$ \\
\hline Satellite & 10.093 & 11.570 & 0.240 & 0.936 \\
Central & 10.316 & 12.024 & 0.215 & 0.795 \\
Yang08 & 10.45 & 12.54 & 0.175 & 0.514 \\
\hline & & & & \\
\hline \hline Sample & $\log M_{0}$ & $\log M_{1}$ & $\alpha$ & $\beta$ \\
\hline Satellite & 10.477 & 11.449 & 0.265 & 1.448 \\
Central & 10.680 & 11.875 & 0.257 & 1.236 \\
Yang12 & 10.36 & 11.06 & 0.27 & 4.34 \\
\hline
\end{tabular}

\subsection{The satellite fraction}

The second quantity we probe is the satellite fraction of galaxies. Since a satellite galaxy resides in a more massive halo than a central galaxy of the same luminosity or stellar mass (e.g. Yang et al. 2003), thus the fraction of satellite galaxies as function of luminosity, $f_{\text {sat }}(L)$, or stellar mass, $f_{\text {sat }}\left(M_{*}\right)$, plays an important role in modelling both the small and large scale clustering of galaxies of a given luminosity/stellar mass (e.g. Jing et al. 1998; Berlind \& Weinberg 2002; Yang et al. 2003; van den Bosch et al. 2007; Yang et al. 2012). The satellite fraction as function of luminosity, $f_{\text {sat }}(L)$, is also important for a proper interpretation of the measurements of galaxy-galaxy lensing signals (e.g. Guzik \& Seliak 2002; Mandelbaum et al. 2006; Yang et al. 2006) and pairwise velocity dispersion of galaxies (e.g. Jing \& Börner 2004; Yang et al.|2004), and to understand the quenching of galaxies (e.g. Bluck et al. 2016; Wang et al. 2017).

Here we estimate $f_{\text {sat }}(L)$ and $f_{\text {sat }}\left(M_{*}\right)$ directly from our matched galaxy-subhalo pairs. In the left-hand panels of Fig. 5] we show $f_{\text {sat }}(L)$ as a function of galaxy luminosity. The results are plotted separately for all (upper panels), red and blue (lower panels) galaxies. Since in our Match2 method the central and satellite galaxies are matched with main halos and subhalos separately, by 

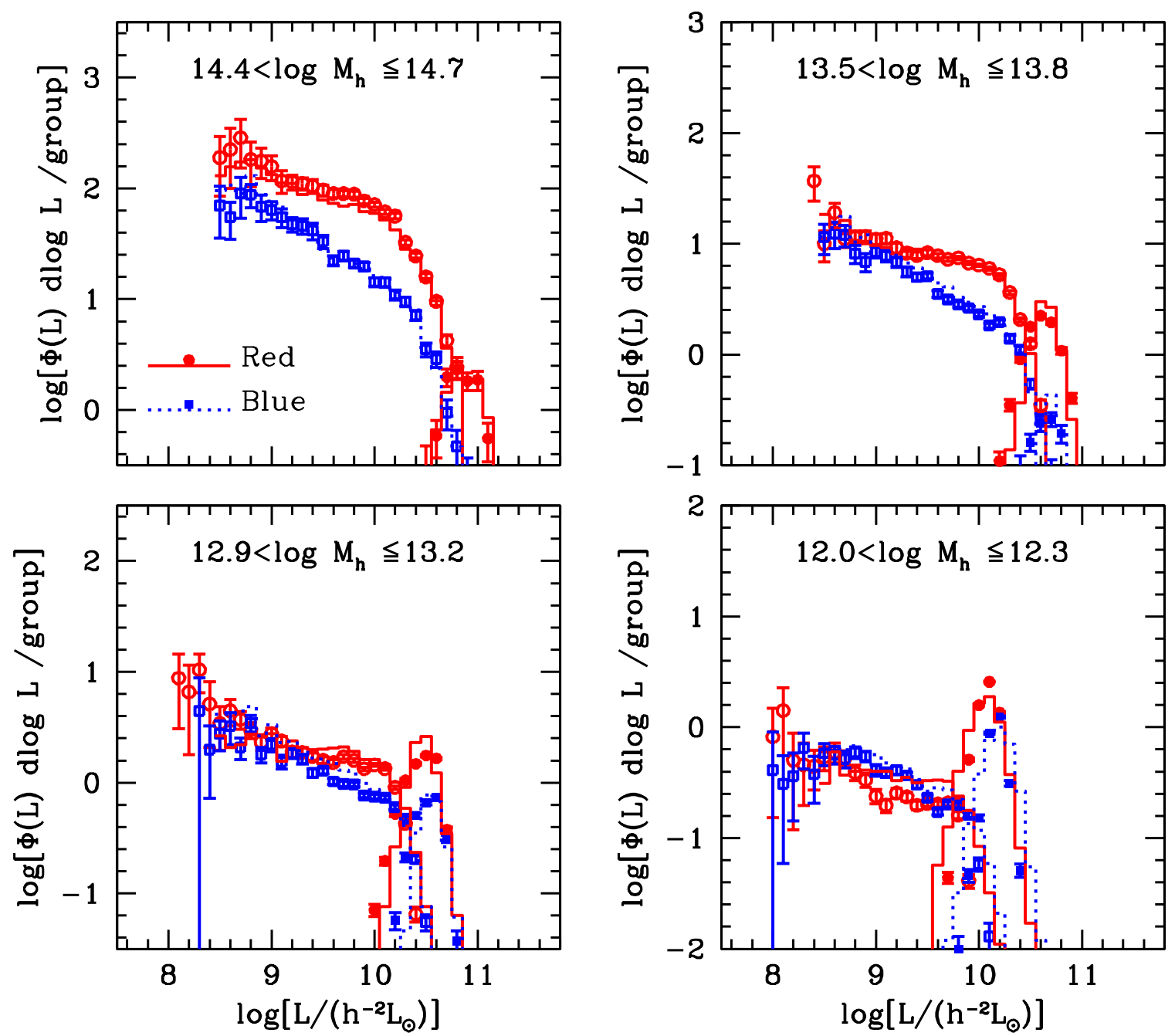

Fig. 7.- Similar to Fig. 6 but here we show the CLFs for red and blue galaxies. In both cases the central and satellite components of the CLFs are indicated separately. Here symbols and lines represent results obtained from SDSS galaxy groups and Match2 method, respectively.

definition, the satellite fractions in our Match2 method follow the same as those of SDSS galaxy groups. We show the resulting satellite fraction in Fig. [5 using solid dots with error bars. Note that, in our probe, we have made use of the modelC sample in Yang et al. (2007), where about $5 \%$ of galaxies that lacking spectroscopic redshifts due to fiber collision effect are assigned with redshifts from their nearest neighbors. As pointed out in Yang et al. (2008), fiber collisions are expected to significantly impact the number of close pairs and hence the satellite fractions $f_{\text {sat }}(L)$. The typical uncertainties induced by adding or removing the fiber collision galaxies are about $5 \%$. Here we adopt this uncertainty value as the error bars shown in Fig. 5 .

First for all galaxies, by comparing the model predictions of the Match1 method with those of Match2 method, we can see, Match1 method predicts roughly consistent satellite fractions for relatively bright galaxies. However, if one goes to fainter galaxies with ${ }^{0.1} \mathrm{M}_{r}-5 \log h>-20.0$, the satellite fractions are significantly underestimated. This discrepancy indicates that the widely used subhalo abundance matching method in literature may not predict the low mass satellite galaxies correctly. One can either add more subhalos (e.g., the disrupted subhalos) in their abundance matching with galaxies, or match central and satellite galaxies separately as we did here in Match2 method.

Next for galaxies that are separated into red and blue populations, compare to those measured from the galaxy groups or Match2 method, the model predictions for Match1 method show much smaller segration between red and blue galaxies. Note that since in our neighborhood abundance matching procedures, we did not make any special treatments between red and blue galaxies, thus the lack of segration for Match1 method is somewhat expected. In general, one may treat red and blue galaxies differently to have a better model prediction of red/blue satellite fractions (e.g. Rodríguez-Puebla et al. 2015), or more straightforwardly by matching central and satellite galaxies separately.

The satellite fraction as a function of galaxy stellar mass are shown in the right panels of Fig. 5. The overall behaviors are quite similar to those shown in the left panels.

Finally, as a comparison, we also show in the lower panels of Fig. 5 the satellite fraction obtained by 

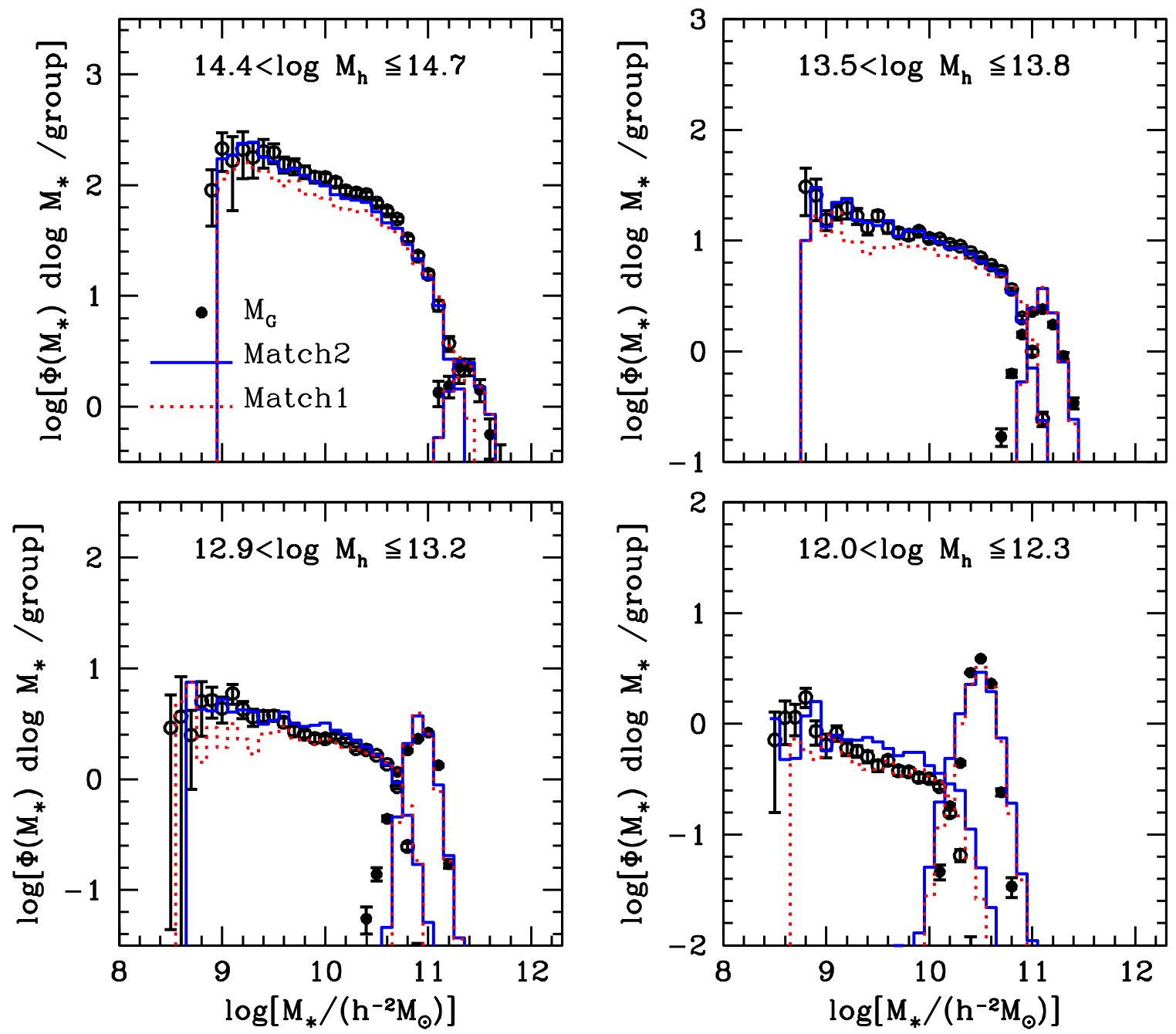

FIG. 8. - The conditional stellar mass functions (CSMFs) of galaxies in groups and host halos of different masses. Symbols correspond to the CSMFs obtained from SDSS galaxy groups, with solid and open circles indicating the contributions from central and satellite galaxies, respectively. The errorbars reflect the 1- $\sigma$ scatter obtained from 200 bootstrap samples. The solid and dotted lines are results obtained for the Match2 and Match1 methods, respectively.

Mandelbaum et al. (2006) for early and late type galaxies (open squares with $95 \%$ confidence level error bars) from the galaxy-galaxy weak lensing measurements. Although their samples are defined differently from ours (early and late types according to galaxy morphologies, v.s. red and blue galaxies according to color), the two measurements agree very well.

\subsection{The conditional luminosity functions}

The conditional luminosity function (CLF) of galaxies in dark halos, $\Phi(L \mid M)$, which describes the average number of galaxies as a function of galaxy luminosity in a dark matter halo of a given mass, plays an important role in our understanding of how galaxies form in dark matter halos (e.g. Yang et al. 2003, 2012, and references therein). Here we directly measure $\Phi(L \mid M)$ from our matched galaxy-subhalo pairs, and compare them to those obtained from the galaxy group catalogs. In order to make proper comparisons, we updated the halo masses of galaxy groups according to the WMAP5 cosmology adopt in this study.

The CLF can be estimated by directly counting galax- ies in halos and groups. However, since the galaxies used for our study are flux limited to $r=17.72$, for a given galaxy luminosity $L$, there is a limiting redshift, $z_{L}$, beyond which galaxies with such a luminosity are not included in the sample. In order to estimate $\Phi(L \mid M)$ at a given $L$, we only use halos and groups that are complete to the redshift limit $z_{L}$. The CLF is obtained by simply counting the average number of galaxies (in luminosity bins) in halos or groups of a given $M$. We show in Fig. 6 the resulting CLFs obtained from galaxy groups of different masses using symbols with error bars, where the error bars are obtained using 200 bootstrap re-samplings of the groups. The contributions of central and satellite galaxies are plotted separately using filled and open symbols, respectively. The solid lines shown in Fig. 6] are the results obtained for our fiducial Match2 method. For comparison, we also show using dotted lines the results obtained for the Match1 method. First, for the central galaxy component, we see that both Match2 and Match1 methods give very similar predictions. According to the comparisons with the data points alone, we see that both Match2 and Match1 methods only agree with data in the 

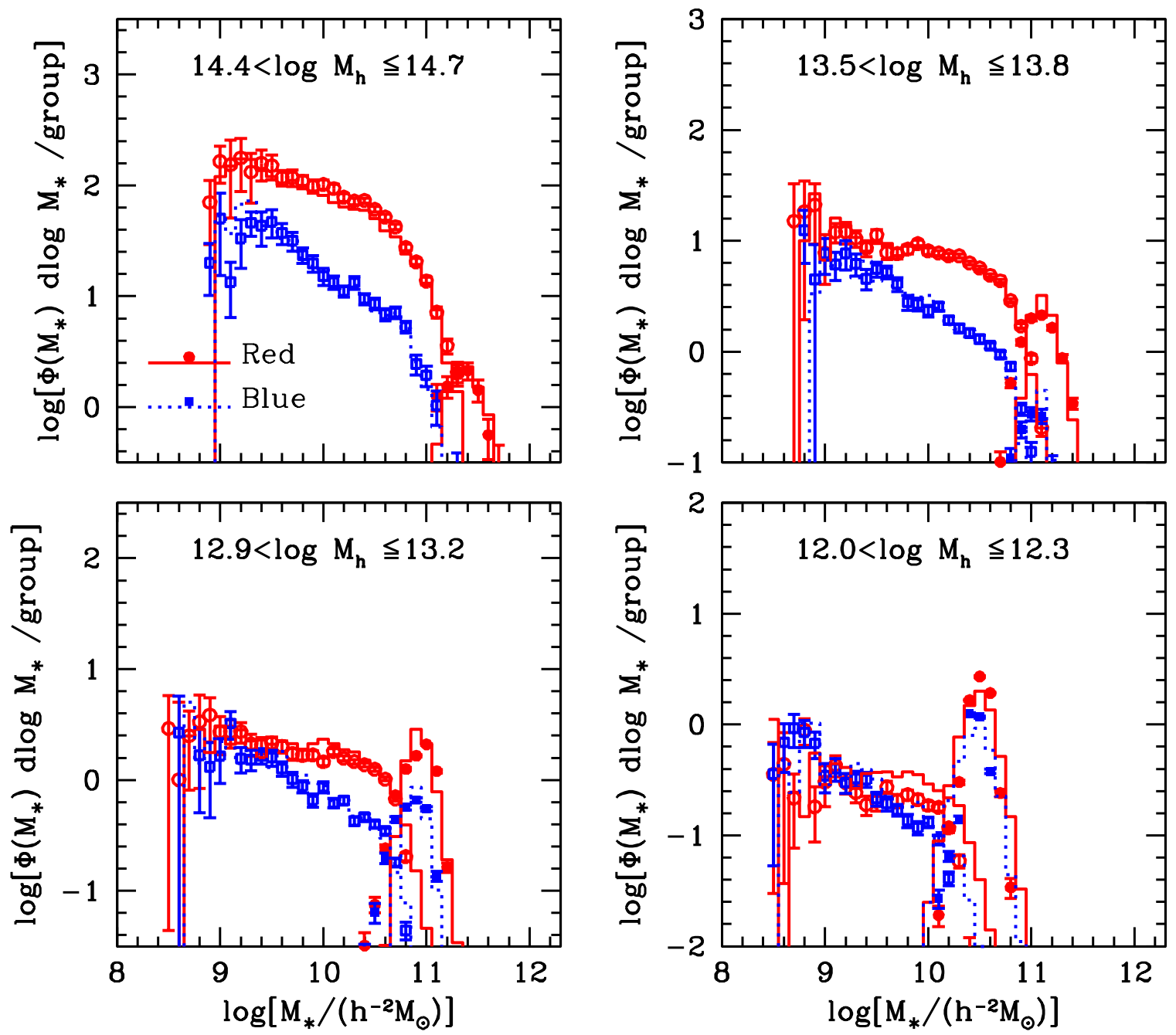

FIG. 9.- Similar to Fig. 8 but here we show the CSMFs for red and blue galaxies. Here symbols and lines represent results obtained from SDSS galaxy groups and Match2 method, respectively.

most massive bin. In all other three halo mass bins, the CLFs of central galaxies show significant deviations. On the other hand however, if we model the CLFs for central galaxies with a lognormal distribution (e.g. Yang et al. 2008),

$$
\Phi_{\text {cen }}\left(L_{c} \mid M\right)=\frac{1}{\sqrt{2 \pi} \sigma_{c}} \exp \left[-\frac{\left(\log L_{c}-\log L\right)^{2}}{2 \sigma_{c}^{2}}\right],
$$

where $L$ is the peak luminosity and $\sigma_{c}$ the lognormal scatter, the discrepancies are indeed not that significant. The two methods both predicted the correct peak luminosities, $L$, of the central galaxies. While the lognormal scatters, $\sigma_{c}$, are slightly smaller in the intermediate halo mass range and slighter larger in the lowest halo mass bin at $\sim 0.01$ levels. Note that since the halo mass estimations in the group catalogs are based on the ranking of characteristic group luminosity/stellar masses, where the central galaxy luminosity/stellar mass and halo mass is somewhat correlated (Yang et al. 2008), the typical uncertainty in the $\sigma_{c}$ constraints is at $\sim 0.02$ (see their Fig. 4). In addition, in the CLF/SHAM modelings, the typical $\sigma_{c}$ assumed in literature also spans a quite large range, $0.15 \sim 0.20$. Thus, for the general behaviors of our CLF model predictions for central galaxies, such amount of differences are expected. Next for the satellite galaxies, comparing to the CLF obtained from galaxy groups, our fiducial Match2 method gives very nice CLF model predictions in halos with mass $\gtrsim 10^{13.5} h^{-1} \mathrm{M}_{\odot}$. While the satellite galaxies for our Match1 method at relatively low mass end are significantly under-predicted. On the other hand, however, in relatively lower mass halos, the situation is quite different. Our fiducial Match2 method over-predicted the CLF at about $\sim 40 \%$ level at $L \sim 10^{9.5} h^{-2} \mathrm{~L}_{\odot}$. While the Match1 method prediction is much better.

Shown in Fig. 7 are the CLFs measured separately for red and blue galaxies, respectively. Symbols with error bars are results obtained from SDSS galaxy groups. The lines are results obtained for our fiducial Match2 method. Even if we did not make special treatments on the color of galaxies for our neighborhood abundance matching, we still find very similar color dependence as the galaxy groups, where massive halos clearly contain more red galaxies than blue galaxies (both centrals and satellites), while the opposite applies to low mass halos. Note that such a halo mass dependence is indeed quite consistent with the halo quenching mechanism (see e.g. 
Wang et al. 2017, and references therein).

\subsection{The conditional stellar mass functions}

Apart from the CLF, which is more observationally related, we can also measure the CSMF of galaxies. The CSMF, $\Phi\left(M_{*} \mid M_{h}\right)$, which describes the average number of galaxies as a function of galaxy stellar mass in a dark matter halo of a given mass, is more straightforwardly related to theoretical predictions of galaxy formation models than the CLF, because the conversion from stellar mass to luminosity in theoretical models requires detailed modeling of the stellar population and dust extinction. The CSMF can be estimated by directly counting the number of galaxies in groups or halos. However, as the galaxies used here are flux limited, here we need to take into account the completeness limits of galaxies as a function of stellar mass as well.

According to van den Bosch et al. (2008), for the stellar masses of the SDSS galaxies, at given redshift $z$, the stellar mass is complete above:

$$
\begin{aligned}
& \log \left[M_{*, \lim } /\left(h^{-2} \mathrm{M}_{\odot}\right)\right]= \\
& \frac{4.852+2.246 \log D_{L}(z)+1.123 \log (1+z)-1.186 z}{1-0.067 z} .
\end{aligned}
$$

Using this relation, we can obtain the redshift completeness limit $z_{M}$ for a given stellar mass $M_{*}$. Similar to the redshift limit for luminosities, here we only use galaxies and groups (halos) that are below redshift limit $z_{M}$ to estimate the CSMF, $\Phi\left(M_{*} \mid M_{h}\right)$. In Fig. 8 we show the resulting CSMFs for groups of different masses using symbols with error bars. The contributions of central and satellite galaxies again are plotted separately using filled and open symbols.

The solid and dashed lines shown in each panel of Fig. 8 are results measured for Match2 and Match1 methods, respectively. Using the SDSS galaxy group results as references, we find that the general behaviors of the model predictions of Match2 and Match1 methods are quite similar to those of the CLFs. The model prediction of Match2 method agrees with that in the SDSS galaxy groups better in massive halos with mass $\gtrsim 10^{13.5} h^{-1} \mathrm{M}_{\odot}$. While the model prediction for Match1 method is better in lower mass halos.

In Fig. 9 we show the CSMFs separately for red and blue galaxies. Symbols with error bars are results obtained from SDSS galaxy groups, while the lines are results obtained for our fiducial Match2 method. Here again, we see that CSMFs for both red and blue galaxies can be well recovered.

\section{THE BIASES OF GALAXIES}

Having checked the performance of our galaxy-subhalo connections established using a neighborhood abundance matching approach in the HOD framework, we proceed to check their performance on larger scales. Note that since galaxies are only slightly moved to match nearby main halos or subhalos, the auto correlation functions of galaxies on large scales will not change significantly. Here we use the cross correlation functions between galaxies and dark matter particles to check if the large scale environment is properly reproduced in the ELUCID simulation.

\subsection{Cross correlation between galaxies and dark matter}

With all the galaxies been linked with subhalos, we proceed to measure the cross correlation function $(\mathrm{CCF})$ between subhalos (galaxies) and dark matter particles,

$$
\xi_{\mathrm{CCF}}(r)=\frac{P_{\mathrm{HD}}(r)}{P_{\mathrm{HR}}(r)}-1,
$$

where $P_{\mathrm{HD}}(r)$ and $P_{\mathrm{HR}}(r)$ are the number of subhalodark matter and subhalo-random pairs, respectively. For our investigations, the number of random points has been set to be the same as the number of dark matter particles within the simulation box. Those points follow a uniform distribution within the simulation volume.

We first measure the CCFs between galaxies (subhalos) and dark matter particles in the ELUCID simulation for overall galaxy population. We divide the galaxies (subhalos) into 6 subsamples within different absolute magnitude bins: $-17.0 \geq{ }^{0.1} \mathrm{M}_{r}-5 \log h>-18.0,-18.0 \geq$ ${ }^{0.1} \mathrm{M}_{r}-5 \log h>-19.0 \ldots-22.0 \geq{ }^{0.1} \mathrm{M}_{r}-5 \log h$. The open squares shown in Fig. 10 are the CCFs measured for galaxies within these absolute magnitude bins for our fiducial Match2 method. The error bars are obtained from 100 jackknife re-samplings of the galaxies. As a reference, we also show the auto correlation function (ACF) of dark matter particles in the ELUCID simulation in each panel of Fig. 10 using a dotted line. Comparing to the ACF of dark matter, the CCFs of galaxies show somewhat weaker and stronger clustering strength for fainter and brighter subsamples, which is qualitatively consistent with those observational measurements of galaxy biases using ACFs (e.g. Zehavi et al. 2005; Wang et al. 2007).

In addition to the $\mathrm{ACF}$ of dark matter particles, for comparison, we also show using solid line the results obtained for the Match1 method. The main difference induced by the Match2 and Match1 methods are the satellite fraction of galaxies, especially at the faint end. According to the $\mathrm{CCF}$ comparison, we see that:

1. The galaxies in the two samples give very similar results on large scales at $r \geq 5 h^{-1} \mathrm{Mpc}$.

2. On small scales, we see Match2 method has stronger clustering strength, especially in the faint galaxy subsamples.

These features indicate that the clustering measurements of galaxies on small scales are also very important for the HOD/CLF modelling, especially in constraining the satellite components.

Apart from the CCFs of overall galaxy population, we also measure the CCFs of galaxies that are separated in to red and blue subsamples. We show in Fig 11 the CCFs measured separately for red blue galaxies for the Match2 method using solid dots and open squares, respectively. The CCFs of red and blue subsamples show quite different behaviors where red galaxies show overall stronger clustering strength than blue galaxies except in the brightest magnitude bin. Note that in our neighbor abundance matching approach, we did not distinguish between red and blue galaxies. Thus the different clustering behaviors of red and blue galaxies are caused by 


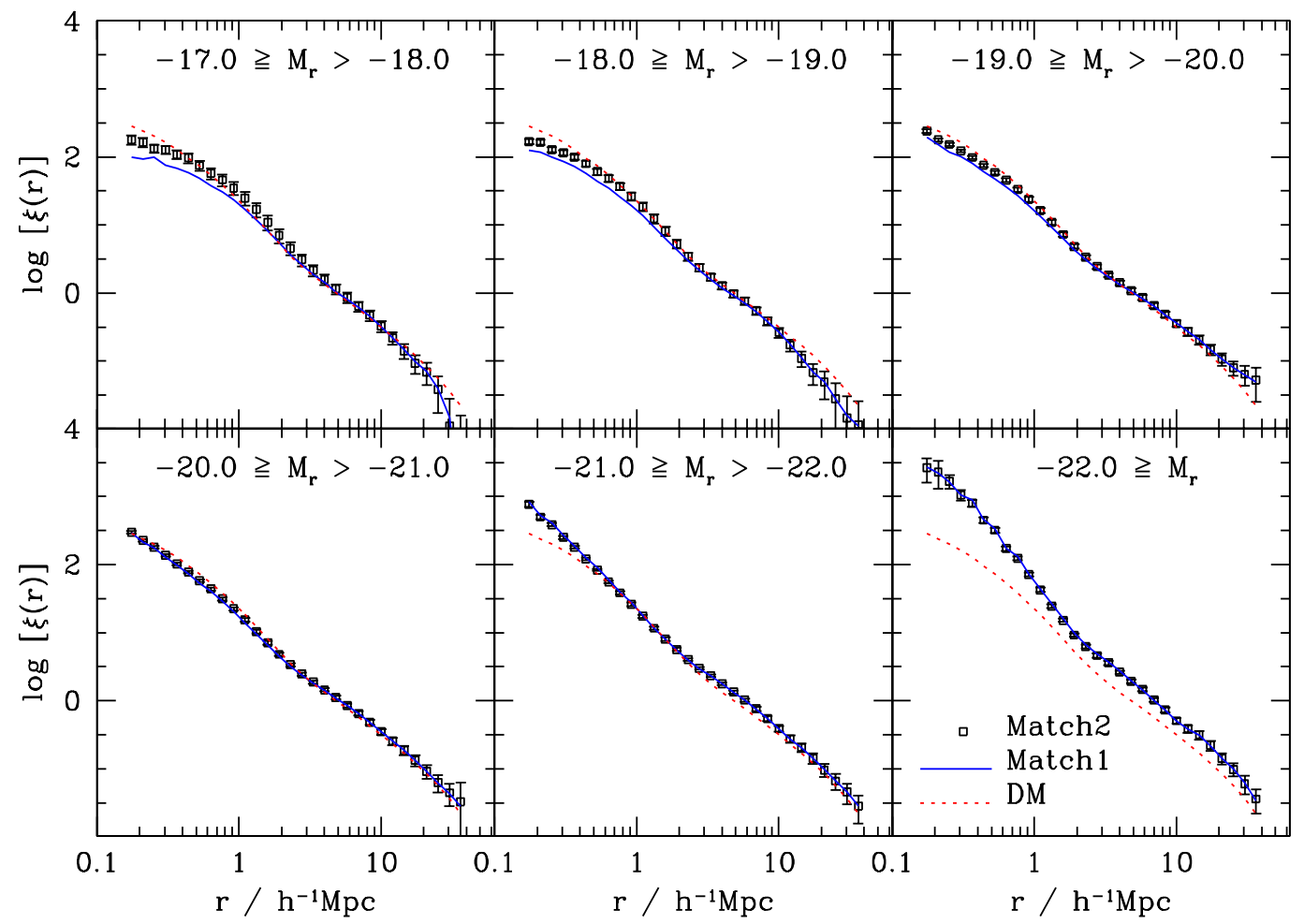

FIG. 10.- Cross correlation functions between galaxies (subhalos) and dark matter particles. Different panels correspond to galaxies in different absolute magnitude bins as indicated. In each panel, the open squares and error bars are results obtained for Match2 method, while the solid line are results obtained for Match1 method. For reference, the dotted lines indicate the dark matter auto correlation in the ELUCID simulation.

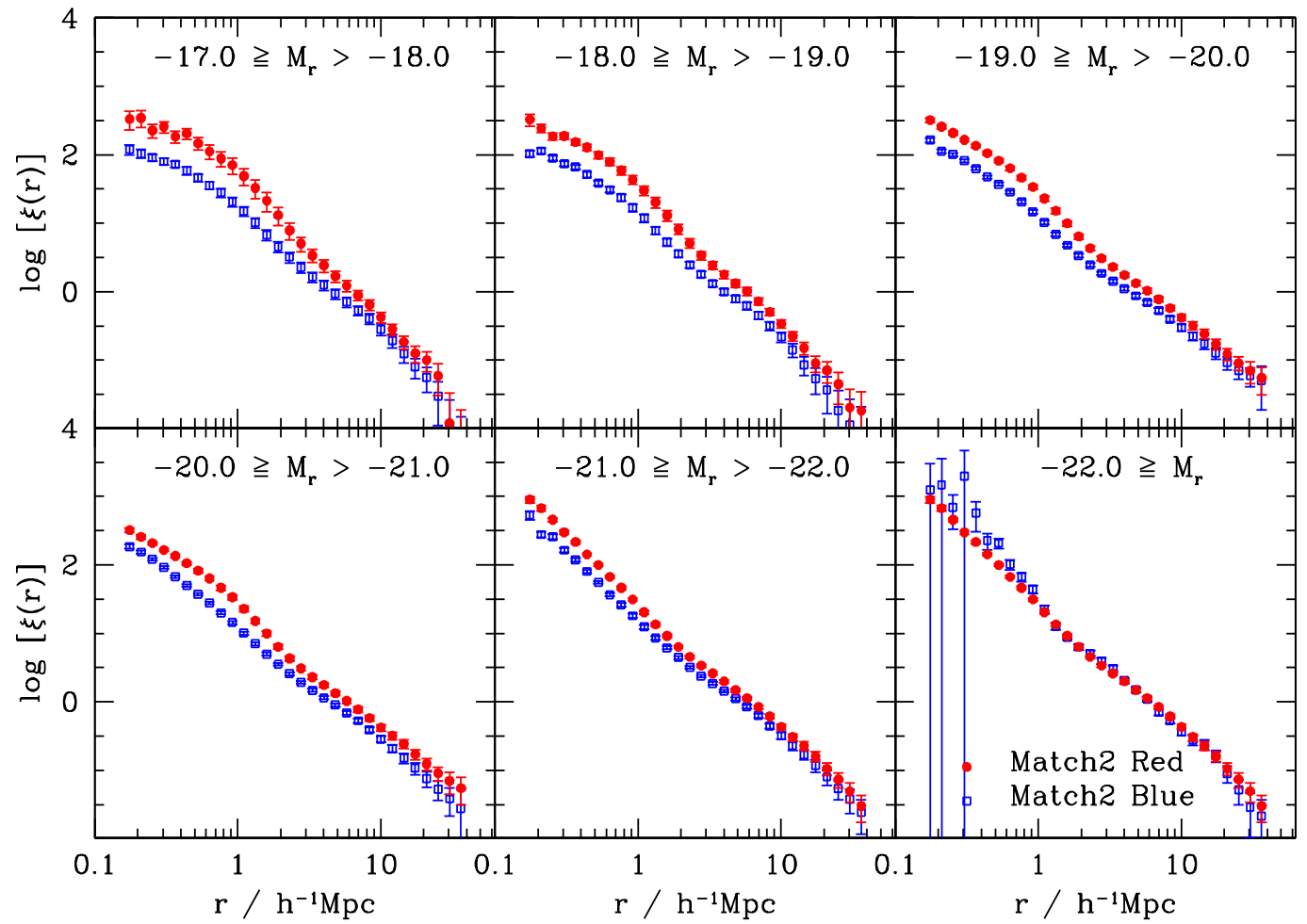

FIg. 11.- Similar to Fig. 10 but here for galaxies (subhalos) that are separated into red and blue subsamples. Here we only show results obtained for Match2 method. 
their large scale environments, e.g., satellite fraction, and host halo masses, etc.

Other than the luminosities of galaxies, we also considered galaxies of different stellar masses. Similar to the treatments for luminosities, we separate galaxies into 6 subsamples within different stellar mass bins: $9.0 \leq$ $\log M_{*}<9.4,9.4 \leq \log M_{*}<9.8 \ldots 11.0 \leq \log M_{*}$. The clustering properties of galaxies in different stellar mass bins are very similar to those in different absolute magnitude bins, which for simplicity, are not shown explicitly here.

To quantify the clustering strengths of galaxies, we show in Fig. 12 the ratios of the galaxy-dark matter CCFs and the dark matter-dark matter ACFs, which are indeed the bias of galaxies as a function of radius. Here results are shown separately for galaxies of different colors and in different absolute magnitude bins as indicated in the plot. The solid line in each panel are results obtained for our Match2 method. For comparison, we also show the resulting biases extracted from the reconstructed real space ACFs of galaxies obtained by Shi et al. (2016) using dots with error bars. In their study, the redshift space distributions of galaxies are mapped to real space by correcting redshift distortions on both small and large scales. Based on thus reconstructed real space distributions of galaxies, Shi et al. (2016) measured the real space ACFs for galaxies in different absolute magnitude bins which are the same as used in this study. The biases of galaxies are then obtained using the square root of the ratios between the ACFs of galaxies and dark matter particles.

By Comparing our model predictions with those obtained by Shi et al. (2016), we find that these two measurements agree quite well, especially for all and blue galaxies. In most cases, the data points agree with each other within 1- $\sigma$ level, except a few slightly larger than 1- $\sigma$ level. While the discrepancies are somewhat larger for red galaxies. There are quite a number of data points that deviate from each other at about 2- $\sigma$ level. Apart from these agreement check, we also find that both of these bias measurements reveal some curvatures in the $-21.0 \geq{ }^{0.1} \mathrm{M}_{r}-5 \log h>-22.0$ magnitude bin. According to the error bars, we believe that the curvature around $1 h^{-1} \mathrm{Mpc}$ which roughly corresponds to a transition scale from 1-halo to 2 -halo term is robust. The curvature at this scale, which is quite different for red and blue galaxies, might be useful for galaxy formation constraints.

The overall agreement of the bias for our galaxysubhalo matched pairs indicate again that our neighborhood abundance matching method works very well and the large scale environments in our ELUCID simulation is quite reliably reproduced.

\section{HOW TO USE THE MATCHED DATA}

Theoretically, if one can provide a perfect link between the observed galaxies and the subhalos in the simulation, one can then use the properties of individual galaxies to constrain galaxy formation models, e.g. via SAMs, etc., to unprecedented precision. The galaxy-subhalo connections obtained in this study from the ELUCID simulation, although not perfect, are already much better than the traditional subhalo abundance matching approach.

With all the above tests on both small (halo-based) and large scales for the feasibility and reliability of our neighborhood abundance matching method, we proceed to provide some suggestions for the use of the matched galaxy-subhalo connections. Here we suggest to divide all the galaxy-subhalo pairs into three categories, (1) halobased pairs, (2) mass and local volume pairs and (3) local volume pairs.

To make this separation, we first extract all the galaxy-subhalo pairs that are either central-main pairs or satellite-subhalo pairs. We show in Fig. 13 the group mass v.s. halo mass for these two kinds of galaxy-subhalo pairs. Shown in the left and right panels are results for the central-main, satellite-subhalo pairs, respectively. For the central-main pairs, although we see there are some pairs quite off from the consistency line which are caused by various reasons, e.g., survy edge effect, mismatch, etc. the vast majority are consistent with each other. For the satellite-subhalo pairs, the situation is somewhat worse. We can see that quite a large fraction of them are quite offset from the consistency line, which are mainly caused by mismatch of satellite galaxie into different host halos. As an illustration, we use two dotted lines $\log M_{G}-\log M_{h}= \pm 0.3$ to separate the galaxy-subhalo pairs. In total, there are 212798 central-main and 43178 satellite-subhalo pairs with $\left|\log M_{G}-\log M_{h}\right| \leq 0.3$. Comparing to the total number of 277139 central-main and 118930 satellite-subhalo pairs, they consist roughly $77 \%$ and $36 \%$ central and satellite population. If we only consider galaxies in halos with mass $\log M_{h} \geq 13.5$, there are about $51 \%$ and $54 \%$ central and satellite population have $\left|\log M_{G}-\log M_{h}\right| \leq 0.3$.

According to the above behaviors of galaxy-subhalo pairs, we separate them into three categories:

- Cat 1 (halo-based pairs): as pointed out in Tweed et al. (2017), the reconstructed simulation can roughly reproduce more than half of the halos with mass $\gtrsim 10^{13.5} h^{-1} \mathrm{M}_{\odot}$ (e.g., with more than half particles in common). Here we select galaxysubhalo pairs that have $\log M_{h} \geq 13.5$ and $s \leq$ $3 h^{-1} \mathrm{Mpc}$ (where $s$ is the galaxy-subhalo pair separation in redshift space), and $\log M_{s h} \geq 11.5$. In total, there are 830 central-main and $755 \overline{7}$ satellitesubhalo pairs fall into this category. In contrast, for the same criteria, there are 13 central-main and 108 satellite-subhalo pairs fall into this category for a rotated version of the ELUCID simulation. For these pairs, we suggest that one can use the related galaxy properties for those individual main or subhalos to evaluate the galaxy properties predicted by SAMs individually.

- Cat 2 (mass and local volume pairs): all the other galaxy-subhalo pairs that have $\left|\log M_{G}-\log M_{h}\right| \leq$ 0.3. There are 211968 central-main and 38195 satellite-subhalo pairs fall into this category. If we only consider galaxies in halos with mass $\log M_{h} \geq$ 13.5, the related numbers are 809 for central-main and 18250 for satellite-subhalo pairs, respectively. For these pairs, we suggest to compare the overall galaxy properties in similar mass halos in the same locate volumes, e.g., within radius $\sim 20 h^{-1} \mathrm{Mpc}$.

- Cat 3 (local volume pairs): all other galaxy-subhalo pairs. For these pairs, one may compare the over- 


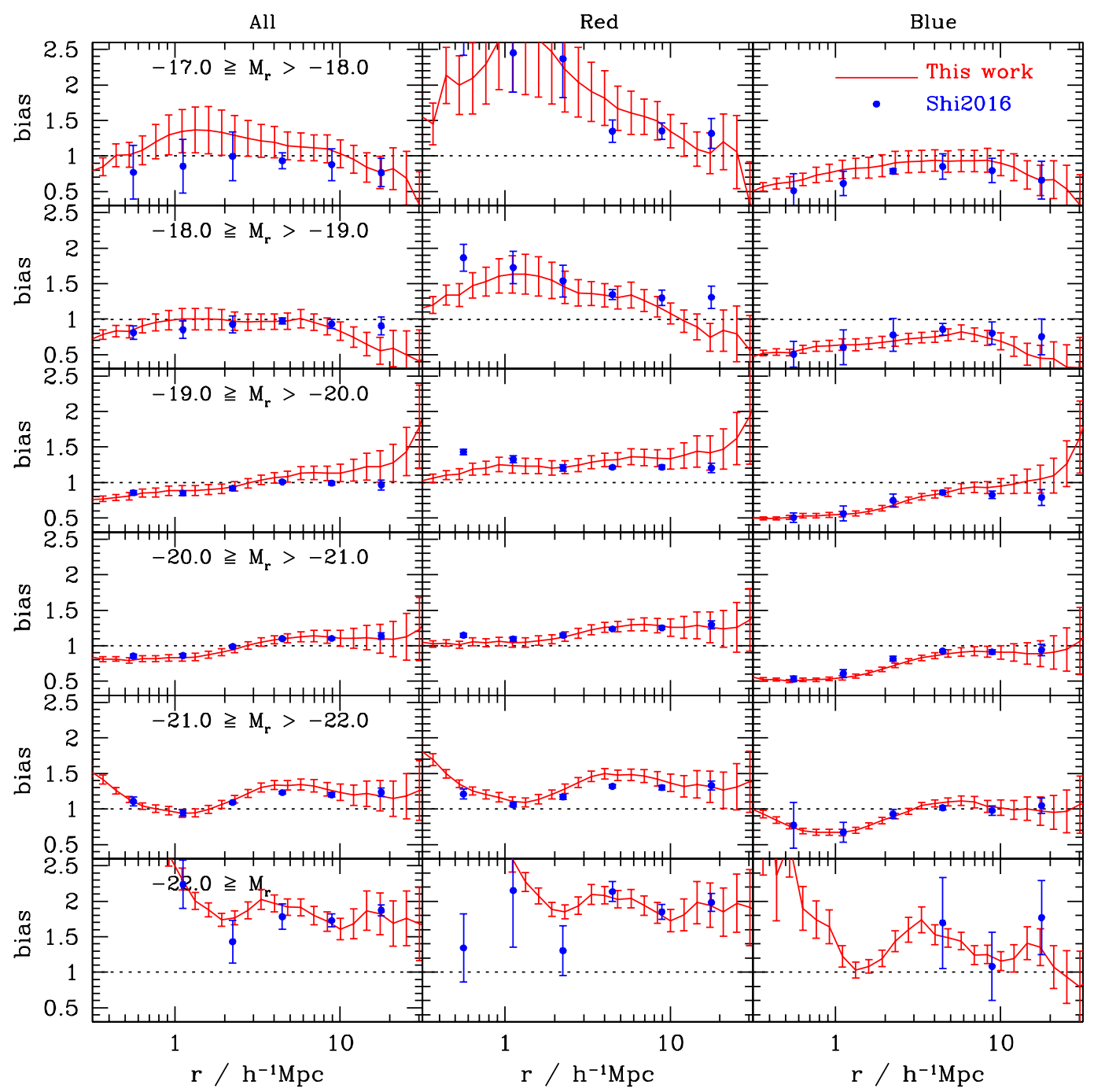

FIG. 12.- The biases for all, red and blue galaxies in different luminosity bins as indicted. In each panel, the biases of galaxies obtained from the CCFs in this study is shown as the solid line with 1- $\sigma$ error bars. For comparison, the dotts with error bars are results obtained by Shi et al. (2016). Here again, we only show results obtained for Match2 method.

all galaxy properties predicted by SAMs in given spherical regions with radius $\sim 20 h^{-1} \mathrm{Mpc}$ with those SDSS galaxies linked with subhalos in the same regions.

Based on these criteria, we will evaluate a few SAMs in a subsequent paper.

\section{SUMMARY}

In this paper, we have proposed a novel neighborhood abundance matching method to link galaxies in the SDSS DR7 observation with dark matter main and subhalos in the ELUCID simulation. Here we used two matching method to make the abundance matching: Match1 is quite popular in SAMs where galaxies are linked to all the survived main halo and subhalos, and Match2 where central galaxies are linked with main halos and satellite galaxies with survived subhalos separately, all of which the maximum masses of the subhalos along their accretion histories are used. We made a list of tests on thus established galaxy-subhalo connections, and the main fea- tures are listed a follows:

- Based on Match2 method, we measured and modelled the luminosity (stellar mass) - subhalo mass relations for central and satellite galaxies separately and found that they have quite different behaviors.

- We have checked the satellite fractions of galaxies as a function of luminosity and stellar mass and found Match1 method somewhat underestimates the related values, especially for low mass galaxies. In addition, unlike the Match2 method, the color segregation of satellite fraction is not well reproduced in Match1 method.

- We have measured the CLFs and CSMFs of galaxies in halos of different masses. Compare to the observational results, the model prediction of Match2 method agrees with that in the SDSS galaxy groups better in massive halos with mass $\gtrsim 10^{13.5} h^{-1} \mathrm{M}_{\odot}$. 

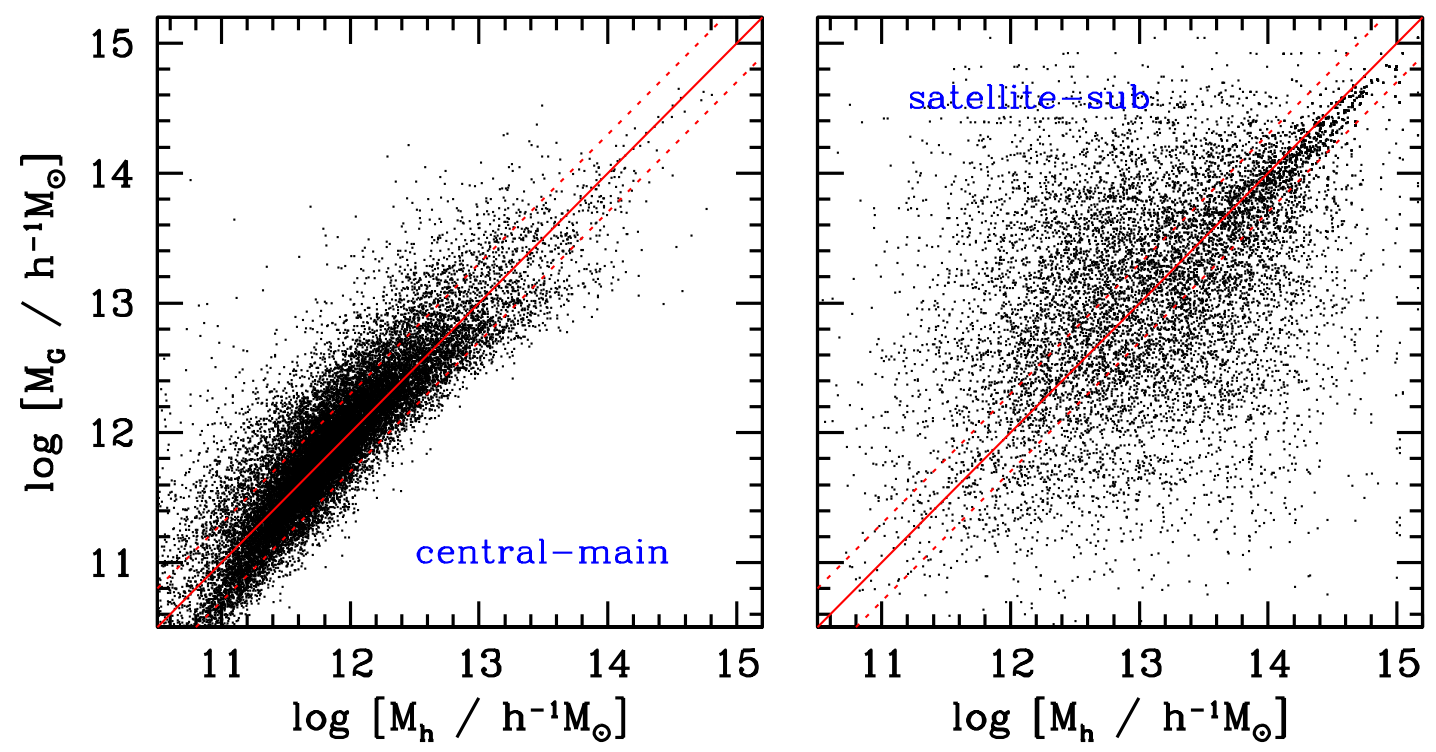

FIG. 13.- The halo mass obtained from galaxy groups $\log M_{G}$ v.s. the host halo mass of the subhalos $\log M_{h}$ in the ELUCID simulation of the matched galaxy-subhalo pairs. Shown in the left and right panels are results for the central-main pairs and satellite-subhalo pairs, respectively.

While the model prediction for Match1 method is better in lower mass halos.

- We have measured the biases of galaxies as a function of radius, which show overall quite nice agreement with the observational results obtained by Shi et al. (2016).

- We have also checked the above quantities separately for red and blue galaxies. All of the results for our Match2 method agree with the direct measurements from observation fairly well.

The above tests show that the Match2 method performs somewhat better than the Match1 method. We thus suggest to make use of the galaxy-subhalo connections established in this sample for galaxy formation studies, e.g. SAMs performed on the ELUCID simulation. In addition, we suggest that those galaxy-subhalo pairs can be divided into three categories: (1) halo-based pairs which can be used to evaluate galaxy properties in individual subhalos, (2) mass and local volume pairs which can be used to evaluate the overall galaxy properties in similar mass halos in the same local volumes, and (3) local volume pairs which can be used to evaluate the overall galaxy properties in the same small volumes. Finally, the galaxy-subhalo links and the subhalo merger trees in the SDSS DR7 region for our ELUCID simulation are available upon request.

\section{ACKNOWLEDGMENTS}

We thank the anonymous referee for helpful comments that greatly improved the presentation of this paper. This work is supported by the 973 Program (No. 2015CB857002), national science foundation of China (grant Nos. 11233005, 11421303, 11522324, 11503064, 11621303, 11733004) and Shanghai Natural Science Foundation, Grant No. 15ZR1446700. We also thank the support of the Key Laboratory for Particle Physics, Astrophysics and Cosmology, Ministry of Education. HJM would like to acknowledge the support of NSFC11673065 and NSF AST-1517528, and FvdB is supported by the US National Science Foundation through grant AST 1516962. WC is supported by the Ministerio de Economía y Competitividad and the Fondo Europeo de Desarrollo Regional (MINECO/FEDER, UE) in Spain through grant AYA2015-63810-P as well as the Consolider-Ingenio 2010 Programme of the Spanish Ministerio de Ciencia e Innovación (MICINN) under grant MultiDark CSD2009-00064.

A computing facility award on the PI cluster at Shanghai Jiao Tong University is acknowledged. This work is also supported by the High Performance Computing Resource in the Core Facility for Advanced Research Computing at Shanghai Astronomical Observatory.

\section{REFERENCES}

Abazajian, K. N., Adelman-McCarthy, J. K., Agüeros, M. A., et al. 2009, ApJS, 182, 543

Avila-Reese, V., \& Firmani, C. 2011, Revista Mexicana de Astronomia y Astrofisica Conference Series, 40, 27

Behroozi, P. S., Conroy, C., \& Wechsler, R. H. 2010, ApJ, 717, 379

Behroozi, P. S., Wechsler, R. H., \& Conroy, C. 2013, ApJ, 770, 57

Bell, E. F., McIntosh, D. H., Katz, N., \& Weinberg, M. D. 2003, ApJS, 149, 289

Berlind A.A., Weinberg D.H., 2002, ApJ, 575, 587
Blanton, M. R., Brinkmann, J., Csabai, I., et al. 2003, AJ, 125, 2348

Blanton, M. R., Schlegel, D. J., Strauss, M. A., et al. 2005, AJ, 129,2562

Blanton, M. R., \& Roweis, S. 2007, AJ, 133, 734

Bluck, A. F. L., Mendel, J. T., Ellison, S. L., et al. 2016,

MNRAS, 462,2559

Bertschinger E., 1987, ApJ, 323, L103

Brown, M. J. I., et al. 2008, ApJ, 682, 937

Bull, P. 2017, MNRAS, 471, 12 
Cacciato M., van den Bosch F.C., More S. Li R., Mo H.J., Yang X., 2009, MNRAS, 394, 929

Cacciato, M., van den Bosch, F. C., More, S., Mo, H., \& Yang, X. 2013, MNRAS, 430, 767

Campbell, D., van den Bosch, F. C., Hearin, A., et al. 2015, MNRAS, 452,444

Cohn, J. D. 2017, MNRAS, 466, 2718

Conroy, C., Wechsler, R. H., \& Kravtsov, A. V. 2006, ApJ, 647, 201

Conroy, C., Gunn, J. E., \& White, M. 2009, ApJ, 699, 486

Contreras, S., Zehavi, I., Baugh, C. M., Padilla, N., \& Norberg, P. 2017, MNRAS, 465, 2833

Cooray, A. 2006, MNRAS, 365, 842

Doumler T., Hoffman Y., Courtois H., Gottlöber S., 2013, MNRAS, 430,888

Dunkley J., et al., 2009, ApJS, 180, 306

Gottloeber S., Hoffman Y., Yepes G., 2010, arXiv, arXiv:1005.2687

Guo, Q., White, S. D. M., Li, C., \& Boylan-Kolchin, M. 2010, MNRAS, 404, 1111

Guzik J., Seljak U., 2002, MNRAS, 335, 311

Hearin, A. P., Zentner, A. R., Berlind, A. A., \& Newman, J. A. 2013, MNRAS, 433, 659

Hoffman Y., Ribak E., 1991, ApJ, 380, L5

Jasche, J., Leclercq, F., \& Wandelt, B. D. 2015, JCAP, 1, 036

Jasche, J., \& Wandelt, B. D. 2013, MNRAS, 432, 894

Jiang, F., \& van den Bosch, F. C. 2016, MNRAS, 458, 2848

Jing Y.P., Mo H.J., Börner G., 1998, ApJ, 494, 1

Jing Y. P., Suto Y., 2002, ApJ, 574, 538

Jing, Y. P., \& Börner, G. 2004, ApJ, 617, 782

Kitaura F. S., Enßlin T. A., 2008, MNRAS, 389, 497

Kitaura F.-S., Gallerani S., Ferrara A., 2012, MNRAS, 420, 61

Klypin A., Hoffman Y., Kravtsov A. V., Gottlöber S., 2003, ApJ, 596,19

Klypin A. A., Shandarin S. F., 1983, MNRAS, 204, 891

Kravtsov A. V., Klypin A., Hoffman Y., 2002, ApJ, 571, 563

Leauthaud, A., Tinker, J., Behroozi, P. S., Busha, M. T., \& Wechsler, R. H. 2011, ApJ, 738, 45

Li, C., Kauffmann, G., Jing, Y. P., et al. 2006, MNRAS, 368, 21

Li, S.-J., Zhang, Y.-C., Yang, X.-H., et al. 2016, Research in Astronomy and Astrophysics, 16, 130

Mandelbaum R., Seljak U., Kauffmann G., Hirata C.M., Brinkmann J., 2006, MNRAS, 368, 715

More, S., van den Bosch, F. C., Cacciato, M., Mo, H. J., Yang, X., \& Li R., 2009, MNRAS, 392, 801

Moster, B. P., Somerville, R. S., Maulbetsch, C., van den Bosch, F. C., Macció, A. V.. Naab, T., \& Oser, L. 2010, ApJ, 710, 903

Moster, B. P., Naab, T., \& White, S. D. M. 2013, MNRAS, 428, 3121

Neistein, E., Weinmann, S. M., Li, C., \& Boylan-Kolchin, M. 2011, MNRAS, 414, 1405

Peacock J.A., Smith R.E., 2000, MNRAS, 318, 1144

Rodríguez-Puebla, A., Avila-Reese, V., Yang, X., et al.

Rodríguez-Puebla, A., Primack, J. R., Avila-Reese, V., \& Faber, S. M. 2017, MNRAS, 470, 651 2015, ApJ, 799, 130

Seljak, U., Aslanyan, G., Feng, Y., \& Modi, C. 2017, JCAP, 12 , 009

Shankar, F., Lapi, A., Salucci, P., De Zotti, G., \& Danese, L. 2006 Ap. 643,14

Sheth R. K., Mo H. J., Tormen G., 2001, MNRAS, 323, 1

Shi, F., Yang, X., Wang, H., et al. 2016, ApJ, 833, 241
Sousa, S. G., Santos, N. C., Adibekyan, V., Delgado-Mena, E., \& Israelian, G. 2015, A\&A, 577, A67

Sousa, S. G., Santos, N. C., Israelian, G., Mayor, M., \& Monteiro, M. J. P. F. G. 2007, A\&A, 469, 783

Springel, V., White, S. D. M., Tormen, G., \& Kauffmann, G. 2001, MNRAS, 328, 726

Springel V. et al., 2005, Nat, 435, 629

Springel V., 2005, MNRAS, 364, 1105

Tinker J.L., Weinberg D.H., Zheng Z., Zehavi I., 2005, ApJ, 631, 41

Tinker, J., Kravtsov, A. V., Klypin, A., et al. 2008, ApJ, 688, 709-728

Tweed, D., Yang, X., Wang, H., et al. 2017, ApJ, 841, 55

Vale, A., \& Ostriker, J. P. 2004, MNRAS, 353, 189

Vale, A., \& Ostriker, J. P. 2006, MNRAS, 371, 1173

van de Weygaert R.. Bertschinger E., 1996, MNRAS, 281, 84

van den Bosch, F. C., Yang, X., \& Mo, H. J. 2003a, MNRAS, 340, 771

van den Bosch, F. C., Yang, X., Mo, H. J., et al. 2007, MNRAS, 376,841

van den Bosch, F. C., Aquino, D., Yang, X., et al. 2008, MNRAS, 387,79

Wang H., Mo H. J., Jing Y. P., Guo Y., van den Bosch F. C.,

Yang X., 2009a, MNRAS, 394, 398

Wang H., Mo H. J., Yang X., van den Bosch F. C., 2012, MNRAS, 420, 1809

Wang H., Mo H. J., Yang X., van den Bosch F. C., 2013, ApJ, $772,63(\mathrm{~W} 13)$

Wang, H., Mo, H. J., Yang, X., Jing, Y. P., \& Lin, W. P. 2014 ApJ, 794, 94

Wang, L., Yang, X., Shen, S., et al. 2014, MNRAS, 439, 611

Wang, H., Mo, H. J., Yang, X., et al. 2016, Ap.J. 831. 164

Wang, H., Mo, H. J., Chen, S., et al. 2017, arXiv:1707.09002

Warren, M. S., Abazajian, K., Holz, D. E., \& Teodoro, L. 2006, ApJ, 646, 881

Wang, Y., Yang, X., Mo, H. J., \& van den Bosch, F. C. 2007, ApJ, 664, 608

White S. D. M., Frenk C. S., Davis M., 1983, ApJ, 274, L1

Yang, X., Mo, H. J., \& van den Bosch, F. C. 2003, MNRAS, 339, 1057

Yang, X., Mo, H. J., Jing, Y. P., van den Bosch, F. C., \& Chu, Y. 2004, MNRAS, 350, 1153

Yang, X., Mo, H. J., van den Bosch, F. C., et al. 2006, MNRAS, 373, 1159

Yang X., Mo H. J., van den Bosch F. C., Pasquali A., Li C., Barden M., 2007, ApJ, 671, 153

Yang, X., Mo, H. J., \& van den Bosch, F. C. 2008, ApJ, 676, 248

Yang, X., Mo, H. J., \& van den Bosch, F. C. 2009, ApJ, 695, 900

Yang, X., Mo, H. J., Zhang, Y., \& van den Bosch, F. C. 2011, ApJ, 741, 13

Yang, X., Mo, H. J., van den Bosch, F. C., Zhang, Y., \& Han, J. 2012, ApJ, 752, 41

York D. G., et al., 2000, AJ, 120, 1579

Zaroubi S., Hoffman Y., Fisher K. B., Lahav O., 1995, ApJ, 449, 446

Zehavi, I., Zheng, Z., Weinberg, D. H., et al. 2005, ApJ, 630, 1

Zheng Z., et al., 2005, ApJ, 633, 791

Zu, Y., \& Mandelbaum, R. 2016, MNRAS, 457, 4360 